\title{
Measurements of the leptonic branching fractions of the tau
}

\author{
DELPHI Collaboration
}

\begin{abstract}
Data collected with the DELPHI detector from 1993 to 1995 combined with previous DELPHI results for data from 1991 and 1992 yield the branching fractions $B(\tau \rightarrow \mathrm{e} \nu \bar{\nu})=\left(17.877 \pm 0.109_{\text {stat }} \pm 0.110_{\text {sys }}\right) \%$ and $B(\tau \rightarrow \mu \nu \bar{\nu})=$ $\left(17.325 \pm 0.095_{\text {stat }} \pm 0.077_{\text {sys }}\right) \%$.
\end{abstract}


P.Abreu $^{21}$, W.Adam ${ }^{50}, \quad$ T.Adye ${ }^{36}, \quad$ P.Adzic ${ }^{11}, \quad$ I.Ajinenko ${ }^{42}, \quad$ Z.Albrecht ${ }^{17}, \quad$ T.Alderweireld ${ }^{2}, \quad$ G.D.Alekseev ${ }^{16}$, R.Alemany ${ }^{49}$, T.Allmendinger ${ }^{17}$, P.P.Allport ${ }^{22}$, S.Almehed ${ }^{24}$, U.Amaldi ${ }^{9}$, N.Amapane ${ }^{45}$, S.Amato ${ }^{47}$, E.G.Anassontzis ${ }^{3}$, P.Andersson ${ }^{44}$, A.Andreazza ${ }^{9}$, S.Andringa ${ }^{21}$, P.Antilogus ${ }^{25}$, W-D.Apel ${ }^{17}$, Y.Arnoud ${ }^{9}$, B.Åsman ${ }^{44}$, J-E.Augustin ${ }^{25}$, A.Augustinus ${ }^{9}$, P.Baillon ${ }^{9}, \quad$ P.Bambade ${ }^{19}, \quad$ F.Barao ${ }^{21}, \quad$ G.Barbiellini ${ }^{46}, \quad$ R.Barbier ${ }^{25}$, D.Y.Bardin ${ }^{16}, \quad$ G.Barker ${ }^{17}$, A.Baroncelli ${ }^{38}$, M.Battaglia ${ }^{15}, \quad$ M.Baubillier ${ }^{23}, \quad$ K-H.Becks ${ }^{52}, \quad$ M.Begalli ${ }^{6}, \quad$ A.Behrmann ${ }^{52}, \quad$ P.Beilliere $^{8}$, Yu.Belokopytov $^{9,53}$, N.C.Benekos ${ }^{31}$, A.C.Benvenuti ${ }^{5}$, C.Berat ${ }^{14}, \quad$ M.Berggren $^{25}$, D.Bertini ${ }^{25}$, D.Bertrand ${ }^{2}$, M.Besancon ${ }^{39}$, F.Bianchi ${ }^{45}$, M.Bigi ${ }^{45}$, M.S.Bilenky ${ }^{16}$, M-A.Bizouard ${ }^{19}$, D.Bloch ${ }^{10}$, H.M.Blom ${ }^{30}$, M.Bonesini ${ }^{27}$, W.Bonivento ${ }^{27}$, M.Boonekamp ${ }^{39}$, P.S.L.Booth ${ }^{22}$, A.W.Borgland ${ }^{4}$, G.Borisov ${ }^{19}$, C.Bosio ${ }^{41}$, O.Botner ${ }^{48}$, E.Boudinov ${ }^{30}$, B.Bouquet $^{19}$, C.Bourdarios ${ }^{19}$, T.J.V.Bowcock ${ }^{22}$, I.Boyko ${ }^{16}$, I.Bozovic ${ }^{11}$, M.Bozzo ${ }^{13}$, P.Branchini ${ }^{38}$, T.Brenke ${ }^{52}$, R.A.Brenner ${ }^{48}$, P.Bruckman ${ }^{18}$, J-M.Brunet ${ }^{8}$, L.Bugge ${ }^{32}$, T.Buran ${ }^{32}$, T.Burgsmueller ${ }^{52}$, B.Buschbeck ${ }^{50}$, P.Buschmann ${ }^{52}$, S.Cabrera ${ }^{49}$, M.Caccia ${ }^{27}$, M.Calvi ${ }^{27}$, T.Camporesi ${ }^{9}$, V.Canale ${ }^{37}$, F.Carena ${ }^{9}, \quad$ L.Carroll $^{22}, \quad$ C.Caso $^{13}$, M.V.Castillo Gimenez ${ }^{49}$, A.Cattai ${ }^{9}$, F.R.Cavallo ${ }^{5}$, V.Chabaud ${ }^{9}$, Ph.Charpentier ${ }^{9}$, L.Chaussard ${ }^{25}$, P.Checchia ${ }^{35}$, G.A.Chelkov ${ }^{16}$, R.Chierici ${ }^{45}$, P.Chliapnikov ${ }^{42}$, P.Chochula ${ }^{7}$, V.Chorowicz ${ }^{25}$, J.Chudoba ${ }^{29}$, K.Cieslik ${ }^{18}$, P.Collins ${ }^{9}$, R.Contri ${ }^{13}$, E.Cortina ${ }^{49}$, G.Cosme ${ }^{19}$, F.Cossutti ${ }^{9}$, J-H.Cowell ${ }^{22}$, H.B.Crawley ${ }^{1}$, D.Crennell ${ }^{36}$, S.Crepe ${ }^{14}$, G.Crosetti ${ }^{13}$, J.Cuevas Maestro ${ }^{33}$, S.Czellar ${ }^{15}$, M.Davenport ${ }^{9}$, W.Da Silva ${ }^{23}$, A.Deghorain ${ }^{2}$, G.Della Ricca ${ }^{46}$, P.Delpierre ${ }^{26}$, N.Demaria ${ }^{9}$, A.De Angelis ${ }^{9}$, W.De Boer ${ }^{17}$, C.De Clercq ${ }^{2}$, B.De Lotto ${ }^{46}$, A.De Min ${ }^{35}$, L.De Paula ${ }^{47}$, H.Dijkstra ${ }^{9}$, L.Di Ciaccio ${ }^{37,9}$, J.Dolbeau ${ }^{8}$, K.Doroba ${ }^{51}$, M.Dracos $^{10}$, J.Drees ${ }^{52}$, M.Dris ${ }^{31}$, A.Duperrin ${ }^{25}$, J-D.Durand ${ }^{9}$, G.Eigen ${ }^{4}$, T.Ekelof ${ }^{48}$, G.Ekspong ${ }^{44}$, M.Ellert ${ }^{48}$, M.Elsing ${ }^{9}$, J-P.Engel ${ }^{10}$, B.Erzen ${ }^{43}$, M.Espirito Santo ${ }^{21}$, E.Falk ${ }^{24}$, G.Fanourakis ${ }^{11}$, D.Fassouliotis ${ }^{11}$, J.Fayot ${ }^{23}$, M.Feindt ${ }^{17}$, P.Ferrari ${ }^{27}$, A.Ferrer ${ }^{49}$, E.Ferrer-Ribas ${ }^{19}$, F.Ferro ${ }^{13}$, S.Fichet ${ }^{23}$, A.Firestone ${ }^{1}$, U.Flagmeyer $^{52}$, H.Foeth ${ }^{9}$, E.Fokitis ${ }^{31}$, F.Fontanelli ${ }^{13}$, B.Franek ${ }^{36}$, A.G.Frodesen ${ }^{4}$, R.Fruhwirth ${ }^{50}$, F.Fulda-Quenzer ${ }^{19}$,

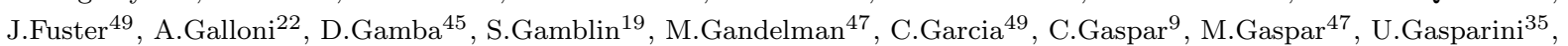
Ph.Gavillet $^{9}$, E.N.Gazis ${ }^{31}$, D.Gele ${ }^{10}$, L.Gerdyukov ${ }^{42}$, N.Ghodbane ${ }^{25}$, I.Gil ${ }^{49}$, F.Glege ${ }^{52}$, R.Gokieli ${ }^{9,51}$, B.Golob ${ }^{43}$, G.Gomez-Ceballos ${ }^{40}$, P.Goncalves ${ }^{21}$, I.Gonzalez Caballero ${ }^{40}$, G.Gopal ${ }^{36}$, L.Gorn ${ }^{1,54}$, M.Gorski ${ }^{51}$, Yu.Gouz ${ }^{42}$, V.Gracco ${ }^{13}$, J.Grahl ${ }^{1}$, E.Graziani ${ }^{38}$, C.Green ${ }^{22}$, H-J.Grimm ${ }^{17}$, P.Gris ${ }^{39}$, G.Grosdidier ${ }^{19}$, K.Grzelak ${ }^{51}$, M.Gunther ${ }^{48}$, J.Guy $^{36}$, F.Hahn ${ }^{9}$, S.Hahn ${ }^{52}$, S.Haider ${ }^{9}$, A.Hallgren ${ }^{48}$, K.Hamacher ${ }^{52}$, J.Hansen ${ }^{32}$, F.J.Harris ${ }^{34}$, V.Hedberg ${ }^{24}$, S.Heising ${ }^{17}$, J.J.Hernandez ${ }^{49}$, P.Herquet ${ }^{2}$, H.Herr ${ }^{9}$, T.L.Hessing ${ }^{34}$, J.-M.Heuser ${ }^{52}$, E.Higon ${ }^{49}$, S-O.Holmgren ${ }^{44}$, P.J.Holt ${ }^{34}$, S.Hoorelbeke ${ }^{2}$, M.Houlden ${ }^{22}$, J.Hrubec ${ }^{50}$, K.Huet ${ }^{2}$, G.J.Hughes ${ }^{22}$, K.Hultqvist ${ }^{44}$, J.N.Jackson ${ }^{22}$, R.Jacobsson ${ }^{9}$, P.Jalocha ${ }^{9}$, R.Janik ${ }^{7}$, Ch.Jarlskog ${ }^{24}$, G.Jarlskog ${ }^{24}$, P.Jarry ${ }^{39}$, B.Jean-Marie ${ }^{19}$, Kristine M.E.Johansen ${ }^{4}$, E.K.Johansson ${ }^{44}$, P.Jonsson ${ }^{25}$, C.Joram ${ }^{9}$, P.Juillot ${ }^{10}$, F.Kapusta ${ }^{23}$, K.Karafasoulis ${ }^{11}$, S.Katsanevas ${ }^{25}$, E.C.Katsoufis ${ }^{31}$, R.Keranen ${ }^{17}$, B.P.Kersevan ${ }^{43}$, B.A.Khomenko ${ }^{16}$, N.N.Khovanski ${ }^{16}$, A.Kiiskinen ${ }^{15}$, B.King ${ }^{22}$, A.Kinvig ${ }^{22}$, N.J.Kjaer ${ }^{30}$, O.Klapp $^{52}$, H.Klein ${ }^{9}$, P.Kluit ${ }^{30}$, P.Kokkinias ${ }^{11}$, M.Koratzinos ${ }^{9}$, V.Kostioukhine ${ }^{42}$, C.Kourkoumelis ${ }^{3}$, O.Kouznetsov ${ }^{39}$, M.Krammer ${ }^{50}$, E.Kriznic ${ }^{43}$, J.Krstic ${ }^{11}$, Z.Krumstein ${ }^{16}$, P.Kubinec ${ }^{7}$, J.Kurowska ${ }^{51}$, K.Kurvinen ${ }^{15}$, J.W.Lamsa ${ }^{1}$, D.W.Lane ${ }^{1}$, P.Langefeld ${ }^{52}$, V.Lapin ${ }^{42}$, J-P.Laugier ${ }^{39}$, R.Lauhakangas ${ }^{15}$, G.Leder ${ }^{50}$, F.Ledroit ${ }^{14}$, V.Lefebure ${ }^{2}$, L.Leinonen ${ }^{44}$, A.Leisos ${ }^{11}$, R.Leitner ${ }^{29}$, G.Lenzen ${ }^{52}$, V.Lepeltier ${ }^{19}$, T.Lesiak ${ }^{18}$, M.Lethuillier ${ }^{39}$, J.Libby ${ }^{34}$, D.Liko ${ }^{9}$, A.Lipniacka ${ }^{44}$, I.Lippi ${ }^{35}$, B.Loerstad ${ }^{24}$, J.G.Loken ${ }^{34}$, J.H.Lopes ${ }^{47}$, J.M.Lopez ${ }^{40}$, R.Lopez-Fernandez ${ }^{14}$, D.Loukas ${ }^{11}$, P.Lutz ${ }^{39}$, L.Lyons ${ }^{34}$, J.MacNaughton ${ }^{50}$, J.R.Mahon ${ }^{6}$, A.Maio ${ }^{21}$, A.Malek ${ }^{52}$, T.G.M.Malmgren ${ }^{44}$, S.Maltezos ${ }^{31}$,

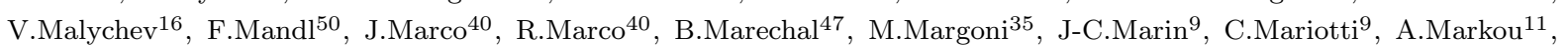
C.Martinez-Rivero ${ }^{19}$, F.Martinez-Vidal ${ }^{49}$, S.Marti i Garcia ${ }^{9}, \quad$ J.Masik $^{12}$, N.Mastroyiannopoulos ${ }^{11}, \quad$ F.Matorras ${ }^{40}$, C.Matteuzzi $^{27}$, G.Matthiae ${ }^{37}, \quad$ F.Mazzucato ${ }^{35}, \quad$ M.Mazzucato $^{35}, \quad$ M.Mc Cubbin ${ }^{22}, \quad$ R.Mc Kay ${ }^{1}, \quad$ R.Mc Nulty ${ }^{22}$, G.Mc Pherson ${ }^{22}$, C.Meroni ${ }^{27}$, W.T.Meyer ${ }^{1}$, A.Miagkov ${ }^{42}$, E.Migliore ${ }^{45}$, L.Mirabito ${ }^{25}$, W.A.Mitaroff ${ }^{50}$, U.Mjoernmark ${ }^{24}$, T.Moa ${ }^{44}$, M.Moch ${ }^{17}$, R.Moeller ${ }^{28}$, K.Moenig ${ }^{9}$, M.R.Monge ${ }^{13}$, X.Moreau ${ }^{23}$, P.Morettini ${ }^{13}$, G.Morton ${ }^{34}$, U.Mueller ${ }^{52}$, K.Muenich $^{52}$, M.Mulders ${ }^{30}$, C.Mulet-Marquis ${ }^{14}, \quad$ R.Muresan ${ }^{24}$, W.J.Murray ${ }^{36}$, B.Muryn ${ }^{14,18}$, G.Myatt ${ }^{34}$, T.Myklebust ${ }^{32}$, F.Naraghi ${ }^{14}$, M.Nassiakou ${ }^{11}$, F.L.Navarria ${ }^{5}$, S.Navas ${ }^{49}$, K.Nawrocki ${ }^{51}$, P.Negri ${ }^{27}$, S.Nemecek ${ }^{12}$, N.Neufeld $^{9}$, N.Neumeister ${ }^{50}$, R.Nicolaidou ${ }^{39}$, B.S.Nielsen ${ }^{28}, \quad$ M.Nikolenko ${ }^{10,16}$, V.Nomokonov ${ }^{15}$, A.Normand $^{22}$, A.Nygren $^{24}$, V.Obraztsov ${ }^{42}$, A.G.Olshevski ${ }^{16}$, A.Onofre ${ }^{21}$, R.Orava ${ }^{15}$, G.Orazi ${ }^{10}, \quad$ K.Osterberg ${ }^{15}, \quad$ A.Ouraou ${ }^{39}$, M.Paganoni ${ }^{27}$, S.Paiano ${ }^{5}$, R.Pain ${ }^{23}$, R.Paiva ${ }^{21}$, J.Palacios ${ }^{34}$, H.Palka ${ }^{18}$, Th.D.Papadopoulou ${ }^{31}$, K.Papageorgiou ${ }^{11}$, L.Pape $^{9}$, C.Parkes ${ }^{9}$, F.Parodi ${ }^{13}$, U.Parzefall ${ }^{22}$, A.Passeri ${ }^{38}$, O.Passon ${ }^{52}$, M.Pegoraro ${ }^{35}$, L.Peralta ${ }^{21}$, M.Pernicka ${ }^{50}$, A.Perrotta $^{5}$, C.Petridou ${ }^{46}$, A.Petrolini ${ }^{13}$, H.T.Phillips ${ }^{36}$, F.Pierre ${ }^{39}$, M.Pimenta $^{21}$, E.Piotto ${ }^{27}$, T.Podobnik $^{43}$, M.E.Pol $^{6}$, G.Polok ${ }^{18}$, P.Poropat ${ }^{46}$, V.Pozdniakov ${ }^{16}$, P.Privitera ${ }^{37}$, N.Pukhaeva ${ }^{16}$, A.Pullia ${ }^{27}$, D.Radojicic ${ }^{34}$, S.Ragazzi ${ }^{27}$, H.Rahmani ${ }^{31}$, P.N.Ratoff ${ }^{20}$, A.L.Read ${ }^{32}, \quad$ P.Rebecchi ${ }^{9}, \quad$ N.G.Redaelli ${ }^{27}, \quad$ M.Regler ${ }^{50}, \quad$ D.Reid ${ }^{30}, \quad$ R.Reinhardt ${ }^{52}$, P.B.Renton ${ }^{34}$, L.K.Resvanis ${ }^{3}$, F.Richard ${ }^{19}$, J.Ridky ${ }^{12}$, G.Rinaudo ${ }^{45}$, O.Rohne ${ }^{32}$, A.Romero ${ }^{45}, \quad$ P.Ronchese ${ }^{35}$, E.I.Rosenberg ${ }^{1}$, P.Rosinsky ${ }^{7}$, P.Roudeau ${ }^{19}$, T.Rovelli ${ }^{5}$, Ch.Royon ${ }^{39}$, V.Ruhlmann-Kleider ${ }^{39}$, A.Ruiz ${ }^{40}$, H.Saarikko ${ }^{15}$, Y.Sacquin $^{39}$, A.Sadovsky ${ }^{16}$, G.Sajot ${ }^{14}$, J.Salt ${ }^{49}$, D.Sampsonidis ${ }^{11}$, M.Sannino ${ }^{13}$, H.Schneider ${ }^{17}$, Ph.Schwemling ${ }^{23}$, B.Schwering ${ }^{52}$, U.Schwickerath ${ }^{17}$, M.A.E.Schyns ${ }^{52}$, F.Scuri ${ }^{46}$, P.Seager ${ }^{20}$, Y.Sedykh ${ }^{16}$, A.M.Segar ${ }^{34}$, R.Sekulin ${ }^{36}$, R.C.Shellard ${ }^{6}$, A.Sheridan ${ }^{22}$, M.Siebel ${ }^{52}$, L.Simard ${ }^{39}$, F.Simonetto ${ }^{35}$, A.N.Sisakian ${ }^{16}$, G.Smadja ${ }^{25}$, O.Smirnova ${ }^{24}$, G.R.Smith ${ }^{36}$, A.Sokolov ${ }^{42}$, O.Solovianov ${ }^{42}$, A.Sopczak ${ }^{17}$, R.Sosnowski ${ }^{51}$, T.Spassov ${ }^{21}$, E.Spiriti ${ }^{38}$, P.Sponholz ${ }^{52}$, S.Squarcia $^{13}$, C.Stanescu ${ }^{38}$, S.Stanic ${ }^{43}$, K.Stevenson ${ }^{34}$, A.Stocchi ${ }^{19}$, J.Strauss ${ }^{50}$, R.Strub ${ }^{10}$, B.Stugu ${ }^{4}$, M.Szczekowski ${ }^{51}$, M.Szeptycka ${ }^{51}$, T.Tabarelli ${ }^{27}$, F.Tegenfeldt ${ }^{48}$, F.Terranova ${ }^{27}$, J.Thomas ${ }^{34}$, J.Timmermans ${ }^{30}$, N.Tinti ${ }^{5}$, L.G.Tkatchev ${ }^{16}$, 


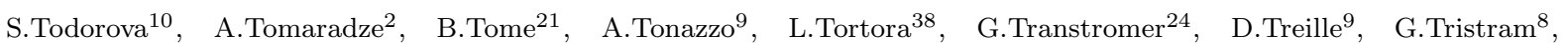
M.Trochimczuk ${ }^{51}$, C.Troncon ${ }^{27}$, A.Tsirou ${ }^{9}$, M-L.Turluer ${ }^{39}$, I.A.Tyapkin ${ }^{16}$, S.Tzamarias ${ }^{11}$, O.Ullaland ${ }^{9}$, V.Uvarov ${ }^{42}$, G.Valenti ${ }^{5}$, E.Vallazza ${ }^{46}$, G.W.Van Apeldoorn ${ }^{30}$, P.Van Dam ${ }^{30}$, W.K.Van Doninck ${ }^{2}$, J.Van Eldik ${ }^{30}$, A.Van Lysebetten ${ }^{2}$, I.Van Vulpen ${ }^{30}$, N.Vassilopoulos ${ }^{34}$, G.Vegni ${ }^{27}$, L.Ventura ${ }^{35}$, W.Venus ${ }^{36,9}$, F.Verbeure ${ }^{2}$, M.Verlato ${ }^{35}$, L.S.Vertogradov ${ }^{16}$, V.Verzi $^{37}$, D.Vilanova ${ }^{39}$, L.Vitale ${ }^{46}$, E.Vlasov ${ }^{42}$, A.S.Vodopyanov ${ }^{16}$, C.Vollmer ${ }^{17}$, G.Voulgaris ${ }^{3}$, V.Vrba ${ }^{12}$, H.Wahlen $^{52}$, C.Walck $^{44}$, C.Weiser ${ }^{17}$, D.Wicke ${ }^{52}$, J.H.Wickens ${ }^{2}$, G.R.Wilkinson ${ }^{9}$, M.Winter ${ }^{10}$, M.Witek ${ }^{18}$, G.Wolf ${ }^{9}$, J.Yi ${ }^{1}$, O.Yushchenko ${ }^{42}$, A.Zalewska ${ }^{18}$, P.Zalewski ${ }^{51}$, D.Zavrtanik ${ }^{43}$, E.Zevgolatakos ${ }^{11}$, N.I.Zimin ${ }^{16,24}$, G.C.Zucchelli ${ }^{44}$, G.Zumerle ${ }^{35}$

${ }^{1}$ Department of Physics and Astronomy, Iowa State University, Ames IA 50011-3160, USA

${ }^{2}$ Physics Department, Univ. Instelling Antwerpen, Universiteitsplein 1, BE-2610 Wilrijk, Belgium and IIHE, ULB-VUB, Pleinlaan 2, BE-1050 Brussels, Belgium

and Faculté des Sciences, Univ. de l'Etat Mons, Av. Maistriau 19, BE-7000 Mons, Belgium

${ }^{3}$ Physics Laboratory, University of Athens, Solonos Str. 104, GR-10680 Athens, Greece

${ }^{4}$ Department of Physics, University of Bergen, Allégaten 55, NO-5007 Bergen, Norway

${ }^{5}$ Dipartimento di Fisica, Università di Bologna and INFN, Via Irnerio 46, IT-40126 Bologna, Italy

${ }^{6}$ Centro Brasileiro de Pesquisas Físicas, rua Xavier Sigaud 150, BR-22290 Rio de Janeiro, Brazil and Depto. de Física, Pont. Univ. Católica, C.P. 38071 BR-22453 Rio de Janeiro, Brazil

and Inst. de Física, Univ. Estadual do Rio de Janeiro, rua São Francisco Xavier 524, Rio de Janeiro, Brazil

${ }^{7}$ Comenius University, Faculty of Mathematics and Physics, Mlynska Dolina, SK-84215 Bratislava, Slovakia

${ }^{8}$ Collège de France, Lab. de Physique Corpusculaire, IN2P3-CNRS, FR-75231 Paris Cedex 05, France

${ }^{9} \mathrm{CERN}, \mathrm{CH}-1211$ Geneva 23, Switzerland

${ }^{10}$ Institut de Recherches Subatomiques, IN2P3 - CNRS/ULP - BP20, FR-67037 Strasbourg Cedex, France

${ }^{11}$ Institute of Nuclear Physics, N.C.S.R. Demokritos, P.O. Box 60228, GR-15310 Athens, Greece

${ }^{12} \mathrm{FZU}$, Inst. of Phys. of the C.A.S. High Energy Physics Division, Na Slovance 2, CZ-180 40, Praha 8, Czech Republic

${ }^{13}$ Dipartimento di Fisica, Università di Genova and INFN, Via Dodecaneso 33, IT-16146 Genova, Italy

${ }^{14}$ Institut des Sciences Nucléaires, IN2P3-CNRS, Université de Grenoble 1, FR-38026 Grenoble Cedex, France

${ }^{15}$ Helsinki Institute of Physics, HIP, P.O. Box 9, FI-00014 Helsinki, Finland

${ }^{16}$ Joint Institute for Nuclear Research, Dubna, Head Post Office, P.O. Box 79, RU-101 000 Moscow, Russian Federation

${ }^{17}$ Institut für Experimentelle Kernphysik, Universität Karlsruhe, Postfach 6980, DE-76128 Karlsruhe, Germany

${ }^{18}$ Institute of Nuclear Physics and University of Mining and Metalurgy, Ul. Kawiory 26a, PL-30055 Krakow, Poland

${ }^{19}$ Université de Paris-Sud, Lab. de l'Accélérateur Linéaire, IN2P3-CNRS, Bât. 200, FR-91405 Orsay Cedex, France

${ }^{20}$ School of Physics and Chemistry, University of Lancaster, Lancaster LA1 4YB, UK

${ }^{21}$ LIP, IST, FCUL - Av. Elias Garcia, 14-1 ${ }^{\circ}$, PT-1000 Lisboa Codex, Portugal

${ }^{22}$ Department of Physics, University of Liverpool, P.O. Box 147, Liverpool L69 3BX, UK

${ }^{23}$ LPNHE, IN2P3-CNRS, Univ. Paris VI et VII, Tour 33 (RdC), 4 place Jussieu, FR-75252 Paris Cedex 05, France

${ }^{24}$ Department of Physics, University of Lund, Sölvegatan 14, SE-223 63 Lund, Sweden

${ }^{25}$ Université Claude Bernard de Lyon, IPNL, IN2P3-CNRS, FR-69622 Villeurbanne Cedex, France

${ }^{26}$ Univ. d'Aix - Marseille II - CPP, IN2P3-CNRS, FR-13288 Marseille Cedex 09, France

${ }^{27}$ Dipartimento di Fisica, Università di Milano and INFN, Via Celoria 16, IT-20133 Milan, Italy

${ }^{28}$ Niels Bohr Institute, Blegdamsvej 17, DK-2100 Copenhagen Ø, Denmark

${ }^{29}$ NC, Nuclear Centre of MFF, Charles University, Areal MFF, V Holesovickach 2, CZ-180 00, Praha 8, Czech Republic

${ }^{30}$ NIKHEF, Postbus 41882, NL-1009 DB Amsterdam, The Netherlands

${ }^{31}$ National Technical University, Physics Department, Zografou Campus, GR-15773 Athens, Greece

${ }^{32}$ Physics Department, University of Oslo, Blindern, NO-1000 Oslo 3, Norway

${ }^{33}$ Dpto. Fisica, Univ. Oviedo, Avda. Calvo Sotelo s/n, ES-33007 Oviedo, Spain

${ }^{34}$ Department of Physics, University of Oxford, Keble Road, Oxford OX1 3RH, UK

${ }^{35}$ Dipartimento di Fisica, Università di Padova and INFN, Via Marzolo 8, IT-35131 Padua, Italy

${ }^{36}$ Rutherford Appleton Laboratory, Chilton, Didcot OX11 OQX, UK

${ }^{37}$ Dipartimento di Fisica, Università di Roma II and INFN, Tor Vergata, IT-00173 Rome, Italy

${ }^{38}$ Dipartimento di Fisica, Università di Roma III and INFN, Via della Vasca Navale 84, IT-00146 Rome, Italy

${ }^{39}$ DAPNIA/Service de Physique des Particules, CEA-Saclay, FR-91191 Gif-sur-Yvette Cedex, France

${ }^{40}$ Instituto de Fisica de Cantabria (CSIC-UC), Avda. los Castros s/n, ES-39006 Santander, Spain

${ }^{41}$ Dipartimento di Fisica, Università degli Studi di Roma La Sapienza, Piazzale Aldo Moro 2, IT-00185 Rome, Italy

${ }^{42}$ Inst. for High Energy Physics, Serpukov P.O. Box 35, Protvino, (Moscow Region), Russian Federation

${ }^{43}$ J. Stefan Institute, Jamova 39, SI-1000 Ljubljana, Slovenia and Laboratory for Astroparticle Physics,

Nova Gorica Polytechnic, Kostanjeviska 16a, SI-5000 Nova Gorica, Slovenia,

and Department of Physics, University of Ljubljana, SI-1000 Ljubljana, Slovenia

${ }^{44}$ Fysikum, Stockholm University, Box 6730, SE-113 85 Stockholm, Sweden

${ }^{45}$ Dipartimento di Fisica Sperimentale, Università di Torino and INFN, Via P. Giuria 1, IT-10125 Turin, Italy

${ }^{46}$ Dipartimento di Fisica, Università di Trieste and INFN, Via A. Valerio 2, IT-34127 Trieste, Italy

and Istituto di Fisica, Università di Udine, IT-33100 Udine, Italy

${ }^{47}$ Univ. Federal do Rio de Janeiro, C.P. 68528 Cidade Univ., Ilha do Fundão BR-21945-970 Rio de Janeiro, Brazil

${ }^{48}$ Department of Radiation Sciences, University of Uppsala, P.O. Box 535, SE-751 21 Uppsala, Sweden

${ }^{49}$ IFIC, Valencia-CSIC, and D.F.A.M.N., U. de Valencia, Avda. Dr. Moliner 50, ES-46100 Burjassot (Valencia), Spain

${ }^{50}$ Institut für Hochenergiephysik, Österr. Akad. d. Wissensch., Nikolsdorfergasse 18, AT-1050 Vienna, Austria

${ }^{51}$ Inst. Nuclear Studies and University of Warsaw, Ul. Hoza 69, PL-00681 Warsaw, Poland

${ }^{52}$ Fachbereich Physik, University of Wuppertal, Postfach 100 127, DE-42097 Wuppertal, Germany

${ }^{53}$ On leave of absence from IHEP Serpukhov

${ }^{54}$ Now at University of Florida 


\section{Introduction}

A fundamental assumption of the Standard Model of electroweak interactions, SM, is universality of the charged and the neutral weak currents. The study of $\tau$ decays at LEP provides a powerful tool for testing this assumption for charged currents. Within the SM the rate for the decay $\tau \rightarrow l \nu_{\tau} \bar{\nu}_{l}$ can be written as [1]:

$$
\Gamma\left(\tau \rightarrow l \nu_{\tau} \bar{\nu}_{l}\right)=\frac{G_{l \tau}^{2} m_{\tau}^{5}}{192 \pi^{3}} f\left(\frac{m_{l}^{2}}{m_{\tau}^{2}}\right) r_{R C}^{\tau}
$$

where it is assumed that the neutrinos are massless. Here, $f\left(\frac{m_{l}^{2}}{m_{\tau}^{2}}\right)$ is a phase space factor with value $f\left(\frac{m_{\mathrm{e}}^{2}}{m_{\tau}^{2}}\right)=1.0000$ and $f\left(\frac{m_{\mu}^{2}}{m_{\tau}^{2}}\right)=0.9726$. The quantity $r_{R C}^{\tau}$ is a factor due to electroweak radiative corrections, which to a good approximation has the value 0.9960, both for $\tau \rightarrow \mathrm{e} \nu \bar{\nu}$ and $\tau \rightarrow \mu \nu \bar{\nu}$ decays. $G_{l \tau}$ is the coupling of the tau to a lepton of type $l$, and equals the Fermi coupling constant if lepton universality holds.

The branching fractions of the decays $\tau \rightarrow \mu \nu \bar{\nu}$ and $\tau \rightarrow \mathrm{e} \nu \bar{\nu}$ can be used to test universality in the couplings of the leptons to the weak charged current by noting that we can write $G_{l \tau}^{2}=\frac{g_{l}^{2} g_{\tau}^{2}}{32 m_{W}^{4}}$ and computing the ratio of the branching ratios into the two final states:

$$
\frac{B(\tau \rightarrow \mu \nu \bar{\nu})}{B(\tau \rightarrow \mathrm{e} \nu \bar{\nu})}=\frac{g_{\mu}^{2}}{g_{e}^{2}} \cdot \frac{f\left(\frac{m_{\mu}^{2}}{m_{\tau}^{2}}\right)}{f\left(\frac{m_{e}^{2}}{m_{\tau}^{2}}\right)} .
$$

Hence, we have a direct comparison between $g_{\mu}$ and $g_{e}$, the couplings of the muon and electron to the charged weak current.

Using the $\tau$ and muon mass and lifetime measurements together with the analogue of equation 1 for muon decay, further universality tests can be performed.

First, eliminating $g_{e}, \tau-\mu$ universality can be tested through the relation:

$$
B(\tau \rightarrow \mathrm{e} \nu \bar{\nu})=\frac{g_{\tau}^{2}}{g_{\mu}^{2}} \cdot\left[\frac{f\left(\frac{m_{e}^{2}}{m_{\tau}^{2}}\right) r_{R C}^{\tau}}{f\left(\frac{m_{e}^{2}}{m_{\mu}^{2}}\right) r_{R C}^{\mu}}\right] \frac{m_{\tau}^{5}}{m_{\mu}^{5}} \frac{1}{\tau_{\mu}} \cdot \tau_{\tau},
$$

where $\tau_{\mu}$ and $\tau_{\tau}$ are $\mu$ and $\tau$ lifetimes. The quantity $r_{R C}^{\mu}$ accounts for electroweak corrections in muon decay, and the factor inside the square brackets equals 1.0004 .

Second, if $g_{\mu}$ is eliminated, $\tau$ - e universality can be tested through the relation:

$$
B(\tau \rightarrow \mu \nu \bar{\nu})=\frac{g_{\tau}^{2}}{g_{e}^{2}} \cdot\left[\frac{f\left(\frac{m_{\mu}^{2}}{m_{\tau}^{2}}\right) r_{R C}^{\tau}}{f\left(\frac{m_{e}^{2}}{m_{\mu}^{2}}\right) r_{R C}^{\mu}}\right] \frac{m_{\tau}^{5}}{m_{\mu}^{5}} \frac{1}{\tau_{\mu}} \cdot \tau_{\tau} .
$$

Here, the factor inside the square brackets equals 0.9731 .

Also to be noted is that the values of $B(\tau \rightarrow \mathrm{e} \nu \bar{\nu})$ and $B(\tau \rightarrow \mu \nu \bar{\nu})$ are necessary inputs to the quantity

$$
R_{\tau}=\frac{\Gamma\left(\tau \rightarrow \nu_{\tau}+\text { hadrons }\right)}{\Gamma(\tau \rightarrow \mathrm{e} \nu \bar{\nu})},
$$

essential for studies of QCD at the $\tau$ mass scale [2]. This offers a further motivation for making these measurements.

Precise measurements of the leptonic branching fractions of the $\tau$ also constrain the $\tau$ neutrino mass, $m_{\nu_{\tau}}[3]$. 
In the following, measurements of $B(\tau \rightarrow \mathrm{e} \nu \bar{\nu})$ and $B(\tau \rightarrow \mu \nu \bar{\nu})$ are presented using data from the DELPHI experiment at LEP collected from 1993 through 1995. Combined with previously published measurements by the DELPHI collaboration [4] using 1991 and 1992 data, numbers on $B(\tau \rightarrow \mathrm{e} \nu \bar{\nu})$ and $B(\tau \rightarrow \mu \nu \bar{\nu})$ based on DELPHI data from 1991 through 1995 are given. These results are then used to test lepton universality. Finally, an estimate of $R_{\tau}$ and a limit on $m_{\nu_{\tau}}$ are given based on these measurements.

\section{Method}

At LEP an abundant supply of $\tau$ leptons is produced through the reaction $\mathrm{e}^{+} \mathrm{e}^{-} \rightarrow$ $\mathrm{Z}^{0} \rightarrow \tau^{+} \tau^{-}$. At these energies, low multiplicity combined with missing energy due to escaping neutrinos cleanly separates the $\tau^{+} \tau^{-}$pairs from other event types, and selection algorithms can be found which are nearly independent of the specific $\tau$ decay mode. Then, the branching fraction for the decay of the $\tau$ to lepton $l$ can be measured using the expression

$$
B\left(\tau \rightarrow l \nu_{\tau} \bar{\nu}_{l}\right)=\frac{N_{l}}{N_{\tau}} \cdot \frac{1-b_{l}}{1-b_{\tau}} \cdot \frac{\epsilon_{\tau}}{\epsilon_{l}},
$$

where $N_{l}$ is the number of identified leptonic decays found in the sample of $N_{\tau} \tau$ candidates, preselected with efficiency $\epsilon_{\tau}$ with a background fraction of $b_{\tau}$. $\epsilon_{l}$ is the total (preselection $\times$ identification) efficiency for selecting a lepton of type $l$, with a background fraction of $b_{l}$. In a $\tau^{+} \tau^{-}$event selection without specific requirements to one of the two $\tau$ candidates in the event, $\epsilon_{\tau}$ will be identical to the $\tau^{+} \tau^{-}$event selection efficiency.

For systematic studies, it is useful to factorise $\epsilon_{l}$ into the product $\epsilon_{l}=\epsilon_{\tau}^{l} \times \epsilon_{l}^{i d}$, where $\epsilon_{\tau}^{l}$ is the efficiency for preselecting a $\tau$ decaying to a lepton of type $l$, and $\epsilon_{i}^{i d}$ is the efficiency for identifying this lepton, measured with respect to the sample of preselected decays. Then the 'bias factor' defined as $\beta_{l}=\epsilon_{\tau} / \epsilon_{\tau}^{l}$ is expected to be close to unity for $\tau^{+} \tau^{-}$ selection algorithms based on purely topological requirements. Several systematic effects on $\epsilon_{\tau}$ might cancel in the ratio $\beta_{l}$. In the expression above, uncertainties due to the $\tau^{+} \tau^{-}$ production cross-section and the integrated luminosity do not enter. This also helps to reduce the systematic uncertainties of the measurements.

The selection procedures were studied using simulated events which were passed through a detailed simulation of the detector response and reconstructed with the same program as the real data. The event generators used were: KORALZ [5] with the TAUOLA 2.5 decay package [6] for $\mathrm{e}^{+} \mathrm{e}^{-} \rightarrow \tau^{+} \tau^{-}$events, DYMU3 [7] for $\mathrm{e}^{+} \mathrm{e}^{-} \rightarrow \mu^{+} \mu^{-}$events, BABAMC [8] for $\mathrm{e}^{+} \mathrm{e}^{-} \rightarrow \mathrm{e}^{+} \mathrm{e}^{-}$, JETSET 7.3 [9] for $\mathrm{e}^{+} \mathrm{e}^{-} \rightarrow \mathrm{q} \overline{\mathrm{q}}$ events and the BDK generator [10] for events with four fermions in the final state, $\mathrm{e}^{+} \mathrm{e}^{-} \mathrm{e}^{+} \mathrm{e}^{-}, \mathrm{e}^{+} \mathrm{e}^{-} \mu^{+} \mu^{-}, \mathrm{e}^{+} \mathrm{e}^{-} \tau^{+} \tau^{-}$and $\mu^{+} \mu^{-} \mu^{+} \mu^{-}$. These events include two photon reactions, and are hereafter called four-fermion events also in the common case when one or two of the final state particles escaped detection. Test samples identified in the data and the use of the redundancy between different components of the detector allowed detailed checks of the simulated detector response. In the case of discrepancies, corrections were derived. With an expected statistical precision well below $1 \%$, these checks are of vital importance in order to keep the systematic uncertainty smaller, and the methods will be detailed below. 


\section{The DELPHI detector}

A detailed description of the DELPHI detector and its performance can be found in [11]. The principal detector components used in this analysis are the tracking devices for charged particle momentum reconstruction, the High Density Projection Chamber (HPC) for electron and photon identification, and the Hadron CALorimeter (HCAL) and muon chambers for muon identification. The main tracking device in DELPHI is the Time Projection Chamber (TPC) which is a large drift chamber extending over the radial distance $35 \mathrm{~cm}<R<111 \mathrm{~cm}$. To enhance the precision of the TPC measurement, tracking is supplemented by a vertex detector (VD), an inner detector (ID) at radii below $35 \mathrm{~cm}$ and the Outer detector (OD) at distances between 197 and $206 \mathrm{~cm}$ from the mean axis. The TPC also provides up to 192 ionisation measurements per charged particle track, useful for electron/hadron separation. The main device for electron identification is the HPC which offers full reconstruction of the longitudinal and transverse components of electromagnetic showers. The HCAL is longitudinally segmented into 20 layers of iron and limited streamer tubes and covers most of the solid angle. The tubes are grouped so that the number of longitudinal segments in the readout is 4 . Between the 18th and 19th HCAL layers and outside the whole calorimeter, there are drift chambers for detecting the muons which are expected to penetrate the whole HCAL.

\section{Preselection of $\mathrm{e}^{+} \mathrm{e}^{-} \rightarrow \boldsymbol{\tau}^{+} \boldsymbol{\tau}^{-}$}

\subsection{Selection criteria}

The reaction $\mathrm{e}^{+} \mathrm{e}^{-} \rightarrow \tau^{+} \tau^{-}$at LEP energies is characterised by two low multiplicity, highly collimated, back-to-back jets of particles, with significant missing energy due to the undetected neutrinos from the two $\tau$ decays. The $\tau^{+} \tau^{-}$event selection described here was common to both leptonic decay channels and very similar to previous studies [12].

Each event was divided into hemispheres by a plane perpendicular to the thrust axis, which was calculated using the charged particles. Each hemisphere had to contain at least one charged particle. The highest momentum charged particle in each hemisphere was defined as the leading particle for that hemisphere. To ensure coverage by the most important detector elements, the TPC, the HPC and the HCAL, the polar angle, $\theta$, of at least one of the two leading particles per event had to satisfy $0.035<|\cos \theta|<0.731$. The point of closest approach of both leading particles from the centre of the interaction region had to be less than $4.5 \mathrm{~cm}$ along the beam direction (the $\mathrm{z}$ axis), and at least one of them had to be within $0.3 \mathrm{~cm}$ in the plane perpendicular to the beam axis (the $R \phi$ plane). These requirements removed most of the background from cosmic rays.

The background from hadronic decays of the $\mathrm{Z}^{0}$ was reduced by requiring no more than six charged particles originating from the interaction region.

Four fermion events and $\mathrm{e}^{+} \mathrm{e}^{-} \rightarrow\left(\mathrm{e}^{+} \mathrm{e}^{-}\right) \mathrm{q} \overline{\mathrm{q}}$ events were rejected by requiring that the isolation angle, defined as the minimum angle between any two charged particles in different hemispheres, had to be greater than $160^{\circ}$. This also gave a further reduction of the $\mathrm{e}^{+} \mathrm{e}^{-} \rightarrow \mathrm{q} \overline{\mathrm{q}}$ background. Isolation angle distributions are shown in fig. 1. Furthermore, since most of the energy in these events is not deposited in the detector, the total energy in the event, $E_{v i s}$, defined as the sum of the energy of the charged particles and the electromagnetic energy unassigned to any charged track, had to be greater than $0.0875 \times E_{c m}$ where $E_{c m}$ is the centre-of-mass energy. For events with only two charged particle tracks 
reconstructed, the absolute value of the vectorial sum of the momenta transverse to the beam axis had to be larger than $0.4 \mathrm{GeV} / c$.

Most of the Bhabha events were excluded by requiring that $E_{\text {rad }}=\sqrt{E_{1}^{2}+E_{2}^{2}} / E_{\text {beam }}$ be less than unity, and the condition $p_{\text {rad }}=\sqrt{p_{1}^{2}+p_{2}^{2}} / p_{\text {beam }}$ being less than unity removed $\mathrm{e}^{+} \mathrm{e}^{-} \rightarrow \mu^{+} \mu^{-}$events as well as most of the remaining Bhabhas. The variables are normalized to the beam energy, $E_{\text {beam }}$, and beam momentum, $p_{\text {beam }}$, respectively. $E_{1}$, $E_{2}$ are the electromagnetic energies deposited in a cone of half-angle $30^{\circ}$ around the leading particle in each hemisphere. The variables $p_{1}, p_{2}$ are the momenta of the leading particle in each hemisphere, in most cases as reconstructed in the tracking devices. An alternative momentum estimate was performed for charged particles having a significant energy deposition in the HPC, consistent with that expected from an electron. This estimator was defined as a weighted average between the momentum from the tracking devices and the energy deposited in the HPC. It was used in the calculation of $p_{\text {rad }}$ whenever the energy deposition in the HPC was at least half the reconstructed momentum and the momentum was larger than $10 \mathrm{GeV} / c$. The $E_{\text {rad }}$ and $p_{\text {rad }}$ distributions are shown in fig. 2.

Finally, in two-prong events, the acollinearity between the two charged particles was required to be greater than $0.5^{\circ}$. This reduced $\mathrm{e}^{+} \mathrm{e}^{-} \rightarrow \mathrm{e}^{+} \mathrm{e}^{-}, \mathrm{e}^{+} \mathrm{e}^{-} \rightarrow \mu^{+} \mu^{-}$and the cosmic background further. The leading particle momentum distribution of the selected tau decay candidates is shown in fig. 3. The disagreement with expectation, assumed to be due to the modelling of the momentum resolution, translates into a systematic uncertainty in the efficiency due to the $p_{\text {rad }}$ requirement, the implications of which are presented in section 4.3. The contributions to the remaining backgrounds are discussed in section 4.2 .

Both decay channels under study depend on the proper functioning of the TPC, the HPC and the HCAL for proper identification. Muon identification requires the proper functioning of the muon chambers in addition. The result is that the $\mathrm{e}^{+} \mathrm{e}^{-} \rightarrow \tau^{+} \tau^{-}$ selection for muon identification is based on a sample corresponding to a slightly lower integrated luminosity than the sample used for electron identification.

The electron identification relies on the HPC, and $\mathrm{e}^{+} \mathrm{e}^{-} \rightarrow \tau^{+} \tau^{-}$candidates were only accepted for electron identification if at least one of the leading particle tracks could be extrapolated to a point on the HPC surface more than $1^{\circ}$ away from the centre of an azimuthal inter-module boundary. This purely geometric requirement results in a slight reduction in the preselection efficiency for electron identification compared to the preselection efficiency of the sample used for muon identification.

Table 1 summarises the results of the $\tau^{+} \tau^{-}$selections for the two modes.

\subsection{Backgrounds in the preselection samples}

The backgrounds in the preselected $\tau^{+} \tau^{-}$samples amount to about $3 \%$, and should be known with a relative precision of $10 \%$ or better to give a systematic error well below the expected statistical precision of the measurements. Predictions from simulation must be checked carefully, as the backgrounds usually come from tails of distributions. Effects of observed discrepancies between simulation and data must be evaluated.

The $\mathrm{e}^{+} \mathrm{e}^{-} \rightarrow \mu^{+} \mu^{-}$and Bhabha backgrounds were measured by fitting these contributions to the $p_{r a d}$ and $E_{r a d}$ distributions respectively, when all other requirements in the selection were applied (Fig. 2). The differences between data and simulation influence the $\tau^{+} \tau^{-}$efficiency as well as the background estimate, and in order to avoid the wrong attribution of efficiency effects to a discrepant background, the range of the fit was cho- 


\begin{tabular}{|l|c|c|}
\hline Decay mode to be identified & $\tau \rightarrow \mu \nu \bar{\nu}$ & $\tau \rightarrow \mathrm{e} \nu \bar{\nu}$ \\
\hline Integrated luminosity $\left(\mathrm{pb}^{-1}\right)$ & 108 & 112 \\
\hline Number of $\tau$ pairs & 68655 & 68668 \\
\hline Efficiency (\%) & $52.57 \pm 0.04$ & $50.87 \pm 0.04$ \\
\hline \hline Backgrounds (\%) & & \\
\hline $\mathrm{e}^{+} \mathrm{e}^{-}$ & $0.80 \pm 0.12$ & $0.74 \pm 0.11$ \\
$\mathrm{q} \overline{\mathrm{q}}$ & $0.68 \pm 0.04$ & $0.69 \pm 0.04$ \\
$\mu^{+} \mu^{-}$ & $0.32 \pm 0.01$ & $0.32 \pm 0.01$ \\
$\mathrm{e}^{+} \mathrm{e}^{-} \mathrm{e}^{+} \mathrm{e}^{-}$ & $0.70 \pm 0.07$ & $0.70 \pm 0.07$ \\
$\mathrm{e}^{+} \mathrm{e}^{-} \tau^{+} \tau^{-}$ & $0.39 \pm 0.04$ & $0.39 \pm 0.04$ \\
$\mathrm{e}^{+} \mathrm{e}^{-} \mu^{+} \mu^{-}$ & $0.26 \pm 0.03$ & $0.26 \pm 0.03$ \\
$\operatorname{cosmics}$ & $0.034 \pm 0.002$ & $0.033 \pm 0.002$ \\
\hline Total & $3.19 \pm 0.15$ & $3.14 \pm 0.15$ \\
\hline
\end{tabular}

Table 1: Summary of $\tau^{+} \tau^{-}$selection statistics. The middle column is the number obtained for extraction of $B(\tau \rightarrow \mu \nu \bar{\nu})$ and the right column is that used for $B(\tau \rightarrow \mathrm{e} \nu \bar{\nu})$. The uncertainties quoted here are from the measurements of the background levels and from simulation statistics. Additional uncertainties are discussed in the text and listed in separate tables for each of the two decay modes.

sen to cover a region dominated by background. The muon pair contribution to $p_{\text {rad }}$ was fitted in the region $1.2<p_{\text {rad }}<1.4$ and had a $\chi^{2}$ of 4.9 for 9 degrees of freedom with an uncertainty in the background of $\pm 3 \%$. Similarly the fit of the Bhabha contribution to $E_{\text {rad }}$ was fitted in the region $1.3<E_{\text {rad }}<1.5$ and had a $\chi^{2}$ of 2.4 for 9 degrees of freedom with an uncertainty of $\pm 7 \%$.

The BABAMC event generator simulates radiative corrections to order $\alpha$. Newer generators, which take into account higher order effects, are presently available. Two of these generators, UNIBAB [14] and BHWIDE [15], were compared to BABAMC and show a significantly larger number of events with small isolation angle and in the low energy tail of the $E_{\text {rad }}$ distribution. After determining the resolution function from fully simulated BABAMC events, a comparison of the three generators was performed for events with an electron identified (sect. 5) in each hemisphere. It was found that the UNIBAB generator produced an enhancement in the $E_{r a d}$ distribution around $E_{r a d}=1$ which was not seen in the data, and it was thus assumed that the background prediciton from UNIBAB would be too high. With the data available, a distinction between BHWIDE and UNIBAB could not be made. Using a sample of events with just one electron identified, it could be determined that BHWIDE predicts $15 \%$ more background than BABAMC. Since BHWIDE is expected to be more accurate than BABAMC, a $15 \%$ upward adjustment of the Bhabha background was made, adding the full adjustment as an additional uncertainty to the Bhabha background.

The amount of $\mathrm{e}^{+} \mathrm{e}^{-} \rightarrow \mathrm{q} \overline{\mathrm{q}}$ background was determined by studying the isolation angle distribution (see fig. 1). Selecting events with charged particle multiplicity of 5 or 6 gave a sample enhanced with $\mathrm{e}^{+} \mathrm{e}^{-} \rightarrow \mathrm{q} \overline{\mathrm{q}}$ events. For this sample of events, the isolation angle distribution was dominated by $\mathrm{e}^{+} \mathrm{e}^{-} \rightarrow \mathrm{q} \overline{\mathrm{q}}$ events for the region between $120^{\circ}$ and $160^{\circ}$, the other main contribution being $\tau^{+} \tau^{-}$events. The amount of $\mathrm{e}^{+} \mathrm{e}^{-} \rightarrow \mathrm{q} \overline{\mathrm{q}}$ background was adjusted to fit the fraction of events observed in this region with respect to the number seen in the region $120^{\circ}$ to $180^{\circ}$. Adjustments of the $\mathrm{e}^{+} \mathrm{e}^{-} \rightarrow \mathrm{q} \overline{\mathrm{q}}$ background of 
$-2 \%$ to $-8 \%$ (depending on the year the data were taken) relative to the estimate using the $\mathrm{e}^{+} \mathrm{e}^{-} \rightarrow \mathrm{q} \overline{\mathrm{q}}$ cross-section were required.

The four-fermion background feeds into the isolation angle distribution from the low side. Without the isolation angle requirement, the leading track momentum distribution of the $\tau^{+} \tau^{-}$sample shows a very large enhancement at low momenta. Most of this is due to $\mathrm{e}^{+} \mathrm{e}^{-} \rightarrow\left(\mathrm{e}^{+} \mathrm{e}^{-}\right) \mathrm{q} \overline{\mathrm{q}}$ events, a background which is negligible in the accepted region, as the isolation angle for these events is generally much smaller than $160^{\circ}$. The verification of the remaining four-fermion background was therefore performed on the sample of identified electrons and muons, and was assumed to hold also for the preselected $\tau^{+} \tau^{-}$sample.

Since no further rejection of four-fermion background was made in the muon analysis, and since the $\mathrm{e}^{+} \mathrm{e}^{-} \rightarrow\left(\mathrm{e}^{+} \mathrm{e}^{-}\right) \mathrm{e}^{+} \mathrm{e}^{-}$suppression performed for the electron analysis (see sect. 6.1) has negligible effect on the final branching fraction estimate, this assumption seems to be well justified. Simulation showed that the contribution from $\mathrm{e}^{+} \mathrm{e}^{-} \rightarrow\left(\mathrm{e}^{+} \mathrm{e}^{-}\right) \tau^{+} \tau^{-}$ events was significant, and had to be accounted for to get a satisfactory description of the final momentum, isolation angle and acollinearity distributions. After an adjustment of each of the three four-fermion background components by $+(10 \pm 10) \%$, overall agreement between data and simulation was found in the preselected sample as well as in the $\tau \rightarrow \mathrm{e} \nu \bar{\nu}$ and $\tau \rightarrow \mu \nu \bar{\nu}$ samples.

The level of cosmic ray events in the sample was estimated by studying the impact parameter distribution. Plotting $r_{1}$ versus $r_{2}$, where $r_{1}$ and $r_{2}$ are the impact parameters of the leading track of each hemisphere, the cosmic ray events are clearly observed as a diagonal band (fig. 4). The density of events in this band was used to estimate the amount of cosmic ray events satisfying the impact parameter requirements.

\subsection{Efficiency of the preselection sample}

Having estimated the backgrounds, the efficiency of a given requirement was checked by comparing the number of events rejected by this requirement in data to the corresponding number from simulation. These numbers were background subtracted to get efficiency estimates, $\epsilon_{d a t a}$ and $\epsilon_{m c}$. The observed differences were taken as estimates of the systematic uncertainties associated to the efficiencies of the requirements. For the isolation angle requirement, only the region with isolation angle larger than $140^{\circ}$ was considered, as the distribution at smaller isolation angles is dominated by $\mathrm{e}^{+} \mathrm{e}^{-} \rightarrow\left(\mathrm{e}^{+} \mathrm{e}^{-}\right) \mathrm{q} \overline{\mathrm{q}}$ events, a background which is not present in the final sample.

As noted in section 2 , it is the way the bias factor, $\beta_{l}$, is affected by an uncertainty in $\epsilon_{\tau}$ which is relevant for the systematic uncertainty in the branching fraction. To estimate the slope of this dependence, the change in $\beta_{l}$ was computed for a given change in $\epsilon_{\tau}$ by varying each requirement around its chosen value. Then the relative systematic uncertainty on $\beta_{l}$ was computed as the product between this slope and the systematic uncertainty in the efficiency as defined above. Table 2 summarizes the results of this study. The resulting systematic uncertainties due to the bias factor are about a quarter of the statistical uncertainty for the $\tau \rightarrow \mathrm{e} \nu \bar{\nu}$ measurement, and just below half of it for the $\tau \rightarrow \mu \nu \bar{\nu}$ case. The larger uncertainty in the $\tau \rightarrow \mu \nu \bar{\nu}$ is mainly due to the $E_{\text {rad }}$ requirement which affects $\tau \rightarrow \mu \nu \bar{\nu}$ decays much less than the other $\tau$ decays, causing a departure from unity of the bias factor, $\beta_{\mu}$, of about $6 \%$.

The systematic uncertainty due to the track impact parameter requirements was determined by varying the requirements with $10 \%$ of their values, and estimating the corresponding changes the branching fraction estimates. Polar and azimuthal angular distributions of the thrust axis were studied to detect possible event losses. A loss of $\tau^{+} \tau^{-}$ 


\begin{tabular}{|l|c|c|c|c|c|}
\hline Quantity & $\epsilon_{\text {data }} / \epsilon_{m c}$ & $\frac{\Delta \beta_{e} / \beta_{e}}{\Delta \epsilon_{\tau} / \epsilon_{\tau}}$ & $\sigma\left(\beta_{e}\right) / \beta_{e}(\%)$ & $\frac{\Delta \beta_{\mu} / \beta_{\mu}}{\Delta \epsilon_{\tau} / \epsilon_{\tau}}$ & $\sigma\left(\beta_{\mu}\right) / \beta_{\mu}(\%)$ \\
\hline$E_{\text {rad }}$ & 0.996 & 0.17 & 0.05 & -0.68 & 0.20 \\
$P_{\text {rad }}$ & 0.992 & -0.11 & 0.07 & 0.04 & 0.02 \\
Isolation angle & 0.994 & 0.08 & 0.05 & -0.27 & 0.16 \\
Visible energy & 0.999 & 0.91 & 0.05 & 1.00 & 0.06 \\
Acolinearity & 0.999 & 0.19 & 0.01 & 0.35 & 0.02 \\
Missing $p_{t}$ & 0.998 & 0.25 & 0.04 & 0.15 & 0.02 \\
\hline Charged track multiplicity & - & - & 0.13 & - & 0.07 \\
Track vertex requirements & - & - & 0.03 & - & 0.06 \\
Possible trigger bias & - & - & 0.007 & - & 0.033 \\
\hline Total systematic & & & 0.18 & & 0.28 \\
\hline
\end{tabular}

Table 2: Dependence of the bias factor, $\beta_{l}$, for a change in efficiency due to a change of requirement in a given variable, and resulting systematic uncertainty on the bias factor. Further systematic uncertainties on $\beta_{l}$ due to the track quality, track multiplicity and trigger requirements.

events in the real data around the azimuthal boundaries between TPC modules was found also in the $\tau \rightarrow \mu \nu \bar{\nu}$ sample, but to a lesser extent in the $\tau \rightarrow \mathrm{e} \nu \bar{\nu}$ sample, hence leading to a possible undescribed bias in that channel. Finally, the multiplicity distribution of $\tau$ decays opposite to a $\tau$ decay with just one charged particle was studied to compare data and simulation in the number of $\tau^{+} \tau^{-}$events rejected due to a total charged particle multiplicity larger than 6 .

While the trigger efficiency to $\tau^{+} \tau^{-}$pairs is very close to unity, a loss of $\mu^{+} \mu^{-}$pairs of $0.23 \%$ has been observed. Clearly, some $\tau^{+} \tau^{-}$with the decay mode $\tau \rightarrow \mu \nu \bar{\nu}$ for both $\tau$-leptons may have been lost too. This affects the bias factors, and the corresponding changes in the branching fraction estimates were included as contributions to the systematic uncertainties.

\section{Analysis of $\tau \rightarrow \mu \nu \bar{\nu}$ decays}

\subsection{Identification requirements}

Muons were selected with very high efficiency by requiring that the muon candidate satisfied at least one of the following conditions: either 1) no single HCAL layer should have more than $3 \mathrm{GeV}$ of deposited energy while the outermost layer should have at least $0.2 \mathrm{GeV}$, or 2) at least two hits in the muon chambers should be associated to the extrapolation of the charged particle track. Both these requirements reject hadrons effectively while keeping most muons. The efficiency of the muon identification drops steeply for momenta below $2 \mathrm{GeV} / c$ as these particles stop before reaching the outer layer of the HCAL. To obtain a uniform and high efficiency it was required that the charged particle momentum should be larger than $0.05 \times p_{\text {beam }}$. It was also required that there should be just one charged particle in the hemisphere. Distributions of the relevant identification variables are shown in fig.5. The implications of the data/simulation discrepancies observed are described in section 5.2 below.

Two further requirements were imposed in order to suppress $\mathrm{e}^{+} \mathrm{e}^{-} \rightarrow \mu^{+} \mu^{-}$events: if a muon was identified in each hemisphere it was required that the total visible energy 
in the event should be less than $0.7 \times$ the centre-of-mass energy. Furthermore, the total energy detected in the hemisphere opposite to the $\tau \rightarrow \mu \nu \bar{\nu}$ candidate should be less than $0.8 \times \mathrm{E}_{\text {beam }}$. To suppress charged hadrons misidentified as muons, it was required that the average energy deposit per HCAL layer be less that $2 \mathrm{GeV}$. Furthermore, as these hadrons are often accompanied by one or more $\pi^{0} \mathrm{~s}$, it was required that the total electromagnetic energy in an $18^{\circ}$ cone around the track should be less than $3 \mathrm{GeV}$.

A total of $21040 \tau \rightarrow \mu \nu \bar{\nu}$ candidates passed all the identification criteria.

\subsection{Efficiency measurements}

The redundancy between the HCAL and the muon chamber identification permits the measurement of the efficiency of one requirement with respect to the other, and corrections to the simulation estimates were performed. The resulting efficiency of the combined requirement was estimated at $(97.72 \pm 0.06) \%$ within the momentum and angular acceptance, and shows good data/simulation agreement (fig. 6). This efficiency estimate is only valid for muons reaching the outer parts of the detector and $\mathrm{e}^{+} \mathrm{e}^{-} \rightarrow \mu^{+} \mu^{-}$events were used to verify the correctness of the simulated identification efficiency when stopping or decaying muons were also accounted for. Good agreement with data was found.

The requirements designed to reduce the pion contamination in the sample made use of the HPC and the HCAL, but not of the muon chambers. A clean sample of about half the $\tau \rightarrow \mu \nu \bar{\nu}$ candidates was therefore selected with the muon chambers and used to measure the efficiency of these background suppression requirements. The efficiency of the multiplicity requirement was also measured using this sample. The precision of these efficiency measurements lead to a systematic uncertainty of $0.2 \%$. Finally, the efficiency of the requirements to remove $\mathrm{e}^{+} \mathrm{e}^{-} \rightarrow \mu^{+} \mu^{-}$events was verified by comparing the number of events rejected in the data with the number rejected by simulation.

The identification efficiency with respect to the preselected sample was estimated at $\epsilon_{i d}^{\mu}=(82.70 \pm 0.20) \%$. Around half of the losses are due to the momentum requirement, while the rest come from the identification and background suppression requirements. The final $\tau \rightarrow \mu \nu \bar{\nu}$ selection efficiency with respect to the full solid angle, $\epsilon_{\mu}$, was $(46.12 \pm$ $0.11) \%$.

\subsection{Background measurements}

Backgrounds from four-fermion final states were determined from simulation and their amount was verified by studying the momentum distribution when the isolation angle requirement was not applied. The $\mu^{+} \mu^{-}$background level was measured by studying the momentum distribution of selected muon candidates when the $p_{\text {rad }}$ requirement was not imposed. The high level of background seen in fig. 7 was well reproduced by simulation, giving confidence that estimates of the much reduced background levels in the final sample were correct within the uncertainties assigned.

The background of $\tau$-leptons decaying to hadrons was measured by selecting $\tau$ decay candidates with one charged particle where the total electromagnetic energy in an $18^{\circ}$ cone around the particle was larger than $3 \mathrm{GeV}$. This selects final states with one or more neutral pions present. This sample of events was subjected to the complete analysis (with the exception of the cone energy requirement), and the remaining sample was used to measure the background from this source. Fig. 8 shows the momentum distribution of the selected sample. After appropriate scaling of the contribution from hadrons the momentum distribution was found to agree well with expectation from simulation. Good 
agreement was also found in the tails of the distributions of the cone energy as well as in the average HCAL energy deposition per layer, after applying the same scale factor to the background contribution to these plots, as shown in fig. 9.

Fig. 10 shows the final momentum distribution compared to expectation. The results of the identification are shown in table 3.

\begin{tabular}{|l|c|}
\hline Number of $\tau \rightarrow \mu \nu \bar{\nu}$ candidates & 21040 \\
\hline Efficiency (\%) & $46.12 \pm 0.11$ \\
\hline \hline Backgrounds (\%) & \\
\hline$\tau$ not decaying to muons & $1.40 \pm 0.08$ \\
$\mu^{+} \mu^{-}$ & $0.33 \pm 0.03$ \\
$\mathrm{e}^{+} \mathrm{e}^{-} \mu^{+} \mu^{-}$ & $1.31 \pm 0.13$ \\
$\mathrm{e}^{+} \mathrm{e}^{-} \tau^{+} \tau^{-}$ & $0.43 \pm 0.04$ \\
$\operatorname{cosmics}$ & $0.17 \pm 0.01$ \\
\hline Total & $3.65 \pm 0.16$ \\
\hline
\end{tabular}

Table 3: Number of $\tau \rightarrow \mu \nu \bar{\nu}$ candidates, selection efficiency and background estimates. The uncertainty in efficiency quoted here is the contribution coming from the identification procedure. Uncertainties in the backgrounds are from simulation and measurements of background levels.

Additional studies were performed to estimate the systematic errors coming from uncertainties on the $\tau$ branching fractions, the $\tau$ polarisation and from the precision of the knowledge of the momentum scale and resolution. The systematic uncertainties are listed in table 4 .

\begin{tabular}{|l|c|}
\hline Systematic uncertainties in $B(\tau \rightarrow \mu \nu \bar{\nu})$ & $(\%)$ \\
\hline Preselection efficiency (i.e. uncertainty in $\left.\beta_{\mu}\right)$ & 0.049 \\
Muon selection efficiency & 0.041 \\
Backgrounds in the muon sample & 0.028 \\
Backgrounds in the preselection sample & 0.027 \\
Uncertainties in the tau branching fractions & 0.004 \\
Momentum scale & 0.003 \\
Scale differences between positive and negative tracks & 0.009 \\
Momentum resolution & 0.006 \\
Uncertainty in polarisation & 0.002 \\
\hline Total systematics & 0.076 \\
\hline
\end{tabular}

Table 4: Summary of the absolute uncertainties on the $\tau \rightarrow \mu \nu \bar{\nu}$ branching fraction measurements. 


\section{Analysis of $\tau \rightarrow \mathrm{e} \nu \bar{\nu}$ decays}

\subsection{Electron identification}

The quantities used for electron identification were the energy loss measurement in the TPC (where a minimum of 38 anode sense wires was required to have a signal recorded), and the ratio, $E / p$, between the electromagnetic energy deposited in the HPC and the momentum reconstructed in the tracking devices. For both these quantities, variables were constructed which were based on the measured value of the variable, its resolution and the value expected for a given particle type. The variables $\Pi_{d E / d x}^{\mathrm{e}(\pi)}$ are defined for the electron and pion hypotheses using the energy loss measurement defined as normalized differences between measurement and expectation:

$$
\Pi_{d E / d x}^{\mathrm{e}(\pi)}=\frac{(d E / d x)_{m e a s}-(d E / d x)_{e x p}^{\mathrm{e}(\pi)}}{\sigma_{d E / d x}},
$$

while $\Pi_{E / p}$ is defined for the electron hypothesis as follows:

$$
\Pi_{E / p}=\frac{(E / p)_{\text {meas }}-(E / p)_{e x p}}{\sigma_{E / p}} .
$$

The inputs to these variables were studied as a function of momentum and of the polar and azimuthal angles of the particle, tuning the distributions in the simulated samples to agree with observation in the data. It was observed that the energy deposition by hadron showers starting before or inside the HPC had to be scaled down by about $10 \%$ in the simulation to get good agreement with data, possibly due to an underestimate of the nuclear interaction length of the material in some of the subdetectors. Such a hypothesis is also consistent with the corrections needed to the levels of backgrounds as estimated from simulation (see discussion in sect. 6.3).

For a particle to be identified as originating from the decay $\tau \rightarrow \mathrm{e} \nu \bar{\nu}$ it had to be the only charged particle in the hemisphere, and have a momentum greater than $0.01 \times p_{\text {beam }}$. All electron candidates were required to have $\Pi_{d E / d x}^{\mathrm{e}}$ greater than -2. For low momentum particles, this rejected hadrons with high power, and no further identification requirement was necessary below $p=0.05 \times p_{\text {beam }}$. For $0.05<p / p_{\text {beam }}<0.5$, two identification requirements could be defined. First, the $\mathrm{dE} / \mathrm{dx}$ information could be used by requiring $\Pi_{d E / d x}^{\pi}>3$. Second, the electromagnetic energy deposition was used by requiring $\Pi_{E / p}>$ -1.5 . To get a very high and uniform efficiency, at least one of these criteria should be fulfilled. For momenta above $0.5 \times p_{\text {beam }}$ it was required that $\Pi_{E / p}$ be larger than -1.5 . Figs. 11 and 12 show the distributions of these identification variables in the relevant momentum ranges.

The residual backgrounds from hadronic $\tau$ decays were reduced by vetoing decays with energy deposited beyond the first layer of the HCAL. Furthermore, a requirment was made on the amount of electromagnetic energy which could not be associated to the charged particle. There should be no additional electromagnetic shower with an energy greater than $4 \mathrm{GeV}$ inside a cone with half-angle of $18^{\circ}$ around the particle trajectory. Additional showers within a $1^{\circ}$ cone around the track and with an azimuthal angle consistent with expectation from bremsstrahlung, were excluded from the calculation of the cone energy.

Electron backgrounds from $\mathrm{e}^{+} \mathrm{e}^{-} \rightarrow\left(\mathrm{e}^{+} \mathrm{e}^{-}\right) \mathrm{e}^{+} \mathrm{e}^{-}$interactions were effectively reduced for two particle events where both momenta were less than $0.2 \times p_{\text {beam }}$ by demanding that the measured $\mathrm{dE} / \mathrm{dx}$ of the charged particle in the opposite hemisphere be inconsistent 
with the value expected for an electron, requiring $\Pi_{d E / d x}^{\pi}<2$. This rejected about two thirds of the remaining $\mathrm{e}^{+} \mathrm{e}^{-} \rightarrow\left(\mathrm{e}^{+} \mathrm{e}^{-}\right) \mathrm{e}^{+} \mathrm{e}^{-}$background.

There were $18273 \tau \rightarrow \mathrm{e} \nu \bar{\nu}$ decays identified. The momentum distribution is found to agree well with expectation from simulation as shown in fig. 13. The identification efficiency and backgrounds are summarised in Table 5, and discussed below.

\subsection{Efficiency measurement}

The redundancy of the $\mathrm{dE} / \mathrm{dx}$ and the $\mathrm{E} / \mathrm{p}$ requirements allows detailed studies of efficiencies and backgrounds in the momentum range $0.05<p / p_{\text {beam }}<0.5$. Fig. 14 a) and b) show the result of such a consistency check for the 1994 data. Corrections were applied bin by bin on the basis of fig. $14 \mathrm{~b}$ ) to get an overall correction to the efficiency. The efficiencies of the $\mathrm{E} / \mathrm{p}$ requirement for momenta above $0.5 \times p_{\text {beam }}$ and the $\Pi_{d E / d x}^{\mathrm{e}}$ requirement were measured using a sample of Bhabha events, and corrections to the simulation results were deduced (fig. $14 \mathrm{c}$ ), separately for each year of data taking. This resolved some discrepancies seen in $\mathrm{B}(\tau \rightarrow \mathrm{e} \nu \bar{\nu})$ when estimates were computed year by year. It was verified that the $\Pi_{d E / d x}^{\mathrm{e}}$ distribution for simulated $\tau \rightarrow \mathrm{e} \nu \bar{\nu}$ decays were compatible with that found for simulated Babhas, as expected since the energy loss from electrons is saturated at the relativistic limit at very low momenta.

A very clean sample of about half the electron candidates was selected by tightening the $\mathrm{dE} / \mathrm{dx}$ requirement to $\Pi_{d E / d x}^{\mathrm{e}}>0$. This sample was used for direct measurements of the efficiencies of the multiplicity requirement as well as the hadron rejection requirements based on the HPC and the HCAL. The precision of these measurements were taken as systematic uncertainties. The branching fraction estimate was not significantly affected by the requirement applied to reject $\mathrm{e}^{+} \mathrm{e}^{-} \rightarrow\left(\mathrm{e}^{+} \mathrm{e}^{-}\right) \mathrm{e}^{+} \mathrm{e}^{-}$, and the full change in the estimate due to applying this requirement was taken as an additional systematic uncertainty of the electron identification efficiency.

The electron identification efficiency, $\epsilon_{l}$, was $(72.30 \pm 0.29) \%$ when measured with respect to the sample of preselected $\tau$ decays. The final $\tau \rightarrow \mathrm{e} \nu \bar{\nu}$ selection efficiency with respect to the full solid angle, $\epsilon_{\mathrm{e}}$, was $(36.79 \pm 0.14) \%$.

\subsection{Background measurements}

The background due to $\mathrm{e}^{+} \mathrm{e}^{-} \rightarrow \mathrm{e}^{+} \mathrm{e}^{-}$and $\mathrm{e}^{+} \mathrm{e}^{-} \rightarrow\left(\mathrm{e}^{+} \mathrm{e}^{-}\right) \mathrm{e}^{+} \mathrm{e}^{-}$events was verified by studying the momentum distribution of identified electrons when requirements designed to reject these backgrounds were not applied. The resulting momentum distributions, (fig. 15) still show reasonable data/simulation agreement, although the background contributions are very enhanced.

For reconstructed momenta below half the beam momentum, the background level from hadrons was estimated by adjusting the tail of the $\Pi_{d E / d x}^{\mathrm{e}}$ to fit the data. For larger momenta, the background contribution to the tail of the $\Pi_{E / p}$ distribution was normalized to the observed data. Both these adjustments led to a significant downscaling of the background relative to the simulation result. Furthermore it was observed that there was less background rejected by the HCAL requirement in simulation compared with data. This can be understood if too few hadrons reach the HCAL in simulation, resulting in more events to be rejected by the TPC and the HPC requirements, exactly as observed. This observation is also consistent with the observation that the simulated average energy deposition from hadrons in the HPC had to be adjusted downwards in order to get agreement in the $\Pi_{E / p}$ distributions. 
The background fraction for the $\tau \rightarrow \mathrm{e} \nu \bar{\nu}$ decay sample, $b_{l}$, was found to be $(5.51 \pm$ $0.41) \%$.

Additional studies were performed to estimate the systematic errors coming from uncertainties on the $\tau$ branching fractions, the $\tau$ polarisation and from the precision of the knowledge of the momentum scale and resolution. The systematic uncertainties are listed in table 6.

\begin{tabular}{|l|c|}
\hline Number of $\tau \rightarrow \mathrm{e} \nu \bar{\nu}$ candidates & 18273 \\
\hline Efficiency $(\%)$ & $36.79 \pm 0.14$ \\
\hline \hline Backgrounds (\%) & \\
\hline$\tau$ not decaying to electrons & $2.11 \pm 0.21$ \\
$\mathrm{e}^{+} \mathrm{e}^{-}$ & $2.20 \pm 0.34$ \\
$\mathrm{e}^{+} \mathrm{e}^{-} \mathrm{e}^{+} \mathrm{e}^{-}$ & $0.79 \pm 0.08$ \\
$\mathrm{e}^{+} \mathrm{e}^{-} \tau^{+} \tau^{-}$ & $0.42 \pm 0.04$ \\
\hline Total & $5.51 \pm 0.41$ \\
\hline
\end{tabular}

Table 5: Number of $\tau \rightarrow \mathrm{e} \nu \bar{\nu}$ candidates, selection efficiency and background estimates.

\begin{tabular}{|l|c|}
\hline Systematic uncertainties in $B(\tau \rightarrow \mathrm{e} \nu \bar{\nu})$ & $(\%)$ \\
\hline Preselection efficiency (i.e. uncertainty in $\left.\beta_{\mathrm{e}}\right)$ & 0.032 \\
Electron selection efficiency & 0.071 \\
Backgrounds in the electron sample & 0.078 \\
Backgrounds in the preselection sample & 0.027 \\
Uncertainties in the tau branching fractions & 0.004 \\
Momentum scale & 0.004 \\
Scale differences between positive and negative tracks & 0.015 \\
Momentum resolution & 0.006 \\
Uncertainty in polarisation & 0.002 \\
\hline Total systematics & 0.115 \\
\hline
\end{tabular}

Table 6: Summary of the absolute uncertainties on the $\tau \rightarrow \mathrm{e} \nu \bar{\nu}$ branching fraction measurement.

\section{$7 \quad$ Results and discussion}

Using data from 1993 to 1995 the following branching fractions were measured:

$$
\begin{aligned}
& B(\tau \rightarrow \mathrm{e} \nu \bar{\nu})=\left(17.947 \pm 0.124_{\text {stat }} \pm 0.115_{\text {sys }}\right) \% \\
& B(\tau \rightarrow \mu \nu \bar{\nu})=\left(17.383 \pm 0.110_{\text {stat }} \pm 0.076_{\text {sys }}\right) \%
\end{aligned}
$$

The result is in agreement with the current world average values [16], and in reasonable agreement with previously published DELPHI results [4] based on data from the years 1991 and 1992. Combining the results obtained here with the results from [4] yields the values:

$$
B(\tau \rightarrow \mathrm{e} \nu \bar{\nu})=\left(17.877 \pm 0.109_{\text {stat }} \pm 0.110_{\text {sys }}\right) \%
$$




$$
B(\tau \rightarrow \mu \nu \bar{\nu})=\left(17.325 \pm 0.095_{\text {stat }} \pm 0.077_{\text {sys }}\right) \%,
$$

which supersede all previously published DELPHI measurements.

A test of e - $\mu$ universality in the weak charged current can be performed using equation 2. From the 93-95 data, the common systematic uncertainty in the two measurements is assumed to be $0.027 \%$, due to the background in the preselection sample. Combined with the statistical anticorrelation between the measurements the total correlation coefficient becomes -0.07 . Accounting for this, the ratio $B(\tau \rightarrow \mu \nu \bar{\nu}) / B(\tau \rightarrow \mathrm{e} \nu \bar{\nu})=0.9686 \pm 0.0098 \pm 0.0072$, is obtained, leading to an estimate of the ratio between the muon and the electron couplings to the charged weak current of $g_{\mu} / g_{e}=0.9979 \pm 0.0063$, for the 93-95 data. Combining this with the estimate $B(\tau \rightarrow \mu \nu \bar{\nu}) / B(\tau \rightarrow \mathrm{e} \nu \bar{\nu})=0.972 \pm 0.017 \pm 0.020$ and $g_{\mu} / g_{e}=1.000 \pm 0.013$ from [4] , the result

$$
\frac{g_{\mu}}{g_{e}}=0.9983 \pm 0.0056
$$

is obtained, consistent with unity.

To test $\tau-\mu$ and $\tau-$ e universality, the published DELPHI value for the $\tau$ lifetime of $(291.4 \pm 3)$ fs [13] is used together with world average values for the lepton masses and the muon lifetime [16]. Then, equations 3 and 4 yield

$$
\begin{aligned}
& \frac{g_{\tau}}{g_{\mu}}=1.0006 \pm 0.0067 \\
& \frac{g_{\tau}}{g_{e}}=0.9987 \pm 0.0062 .
\end{aligned}
$$

The largest contributions to the uncertainty in these estimates $( \pm 0.0052)$ is due to the lifetime measurement, while the branching fraction measurements contribute with \pm 0.0043 and \pm 0.0035 respectively. A more precise DELPHI measurement of the $\tau$ lifetime, based on the full LEP-1 data sample will be published soon, with updated universality tests.

Under the assumption of e- $\mu$ universality, $g_{\mu}=g_{e} \equiv g_{\mathrm{e}, \mu}$, it is possible to give a more stringent test of universality of the coupling of the $\tau$ and that of the two lighter leptons. We combine the two measurements into one leptonic branching fraction, $B_{e, \mu}$, correcting for the phase space suppression of $B(\tau \rightarrow \mu \nu \bar{\nu})$ :

$$
B_{e, \mu}=\left(17.838 \pm 0.066_{\text {stat }} \pm 0.068_{\text {sys }}\right) \% .
$$

to compare the $\tau$ charged current coupling to that of the two lighter leptons. The result

$$
\frac{g_{\tau}}{g_{\mathrm{e}, \mu}}=0.9995 \pm 0.0058
$$

is obtained, in excellent agreement with $\tau$ - $\mu$-e universality.

An estimate of $R_{\tau}$ as defined in equation 5 can be made by computing

$$
R_{\tau}=\frac{1-1.9726 B_{e, \mu}}{B(\tau \rightarrow \mathrm{e} \nu \bar{\nu})} .
$$

The result obtained is:

$$
R_{\tau}=3.626 \pm 0.033
$$

Finally, using our measured value of $B_{\mathrm{e}, \mu}$ together with world averages of other quantities involved, a limit on the $\tau$ neutrino mass can be derived. Using relations given in [3], the value $m_{\nu_{\tau}}^{2}=(-1.48 \pm 2.69) \times 10^{-3}\left(\mathrm{GeV} / c^{2}\right)^{2}$ is obtained. The $1.64 \sigma$ upper limit 
on this number corresponds to a $95 \%$ C.L. upper limit of $m_{\nu_{\tau}}<54 \mathrm{MeV} / c^{2}$. Noting that the central value is unphysical, a more conservative $95 \%$ C.L. upper limit is

$$
m_{\nu_{\tau}}<66 \mathrm{MeV} / c^{2}
$$

which is obtained by shifting the central value to $m_{\nu_{\tau}}=0$.

\section{Acknowledgements}

We are greatly indebted to our technical collaborators, to the members of the CERNSL Division for the excellent performance of the LEP collider, and to the funding agencies for their support in building and operating the DELPHI detector.

We acknowledge in particular the support of Austrian Federal Ministry of Science and Traffics, GZ 616.364/2-III/2a/98,

FNRS-FWO, Belgium, FINEP, CNPq, CAPES, FUJB and FAPERJ, Brazil, Czech Ministry of Industry and Trade, GA CR 202/96/0450 and GA AVCR A1010521, Danish Natural Research Council,

Commission of the European Communities (DG XII),

Direction des Sciences de la Matière, CEA, France,

Bundesministerium für Bildung, Wissenschaft, Forschung und Technologie, Germany,

General Secretariat for Research and Technology, Greece,

National Science Foundation (NWO) and Foundation for Research on Matter (FOM), The Netherlands,

Norwegian Research Council,

State Committee for Scientific Research, Poland, 2P03B06015, 2P03B03311 and SPUB/P03/178/98,

JNICT-Junta Nacional de Investigação Científica e Tecnológica, Portugal,

Vedecka grantova agentura MS SR, Slovakia, Nr. 95/5195/134,

Ministry of Science and Technology of the Republic of Slovenia,

CICYT, Spain, AEN96-1661 and AEN96-1681,

The Swedish Natural Science Research Council,

Particle Physics and Astronomy Research Council, UK,

Department of Energy, USA, DE-FG02-94ER40817. 


\section{References}

[1] W. Marciano and A. Sirlin, Phys. Rev. Lett. 61 (1988) 1815.

[2] E. Braaten, S. Narison and A. Pich, Nucl. Phys. B373 (1992) 209.

[3] J. Swain and L. Taylor, Phys. Rev. D55 1R, 1997.

[4] DELPHI Coll., P. Abreu et al., Phys. Lett. B 357 (1995) 715.

[5] S. Jadach, J.H. Kühn, Z. Was, Comp. Phys. Comm. 79 (1994) 503.

[6] S. Jadach, Z. Was, R. Decker and J.H. Kühn, Comp. Phys. Comm. 76 (1993) 361.

[7] J.E. Campagne and R. Zitoun, Z. Phys. C43 (1983) 469.

[8] F.A. Berends, W. Hollik and R. Kleiss, Nucl. Phys. B304 (1988) 712.

[9] T. Sjöstrand, Comp. Phys. Comm. 82 (1994) 74.

[10] F. A. Berends, P. H. Daverveldt, R. Kleiss, Comp. Phys. Comm. 40 (1986) 271-284, 285-307, 309-326.

[11] DELPHI Coll., P.Aarnio et al., Nucl. Instr. and Meth. A303 (1991) 233.

DELPHI Coll., P.Abreu et al., Nucl. Instr and Meth. A378 (1996) 57.

[12] DELPHI Coll., P.Abreu et al., Nucl. Phys. B417 (1994) 3.

[13] DELPHI Coll., P.Abreu et al., Phys. Lett. B365 (1996) 448.

[14] H. Anlauf et al. report IKDA 95/34, hep-ex/9512006 (unpublished).

[15] S. Jadach et al., Phys. Lett. B390 (1997) 298.

[16] Particle Data Group, Eur. Phys. J. C 3, (1998) 1. 

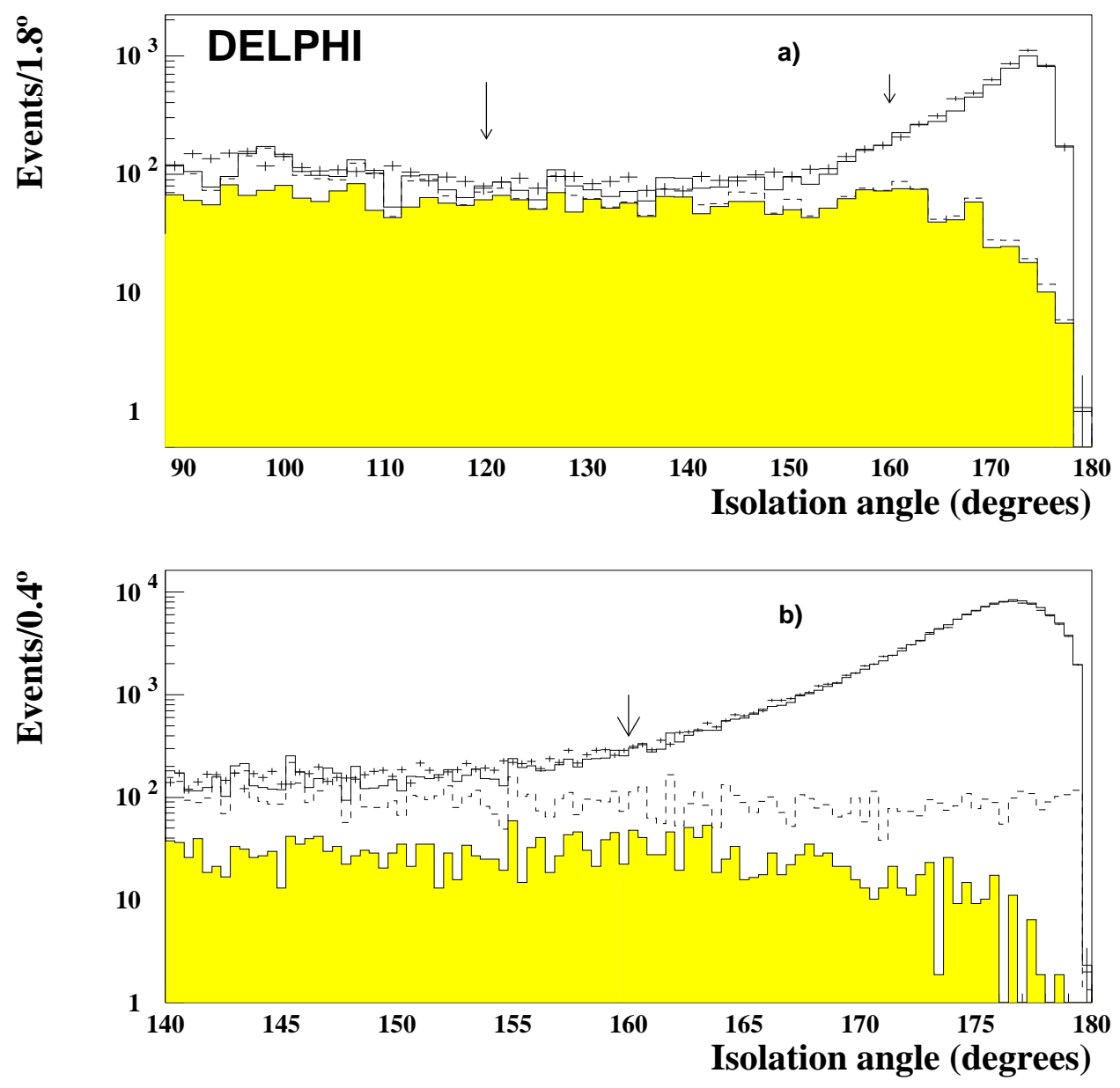

Figure 1: Distribution of the isolation angle when this requirement is not applied. The figure shows the preselected $\tau^{+} \tau^{-}$sample used for electron identification: a) for events with charged particle multiplicity of five or six and b) for all preselected events. The arrows show the range used to normalise the q $\bar{q}$ background. Events to the left of the arrow were rejected. In both figures, crosses show the data, the full line the expectation from simulation, while the dashed line is the expected total background contribution. The shaded histogram shows the $q \bar{q}$ background contribution. 

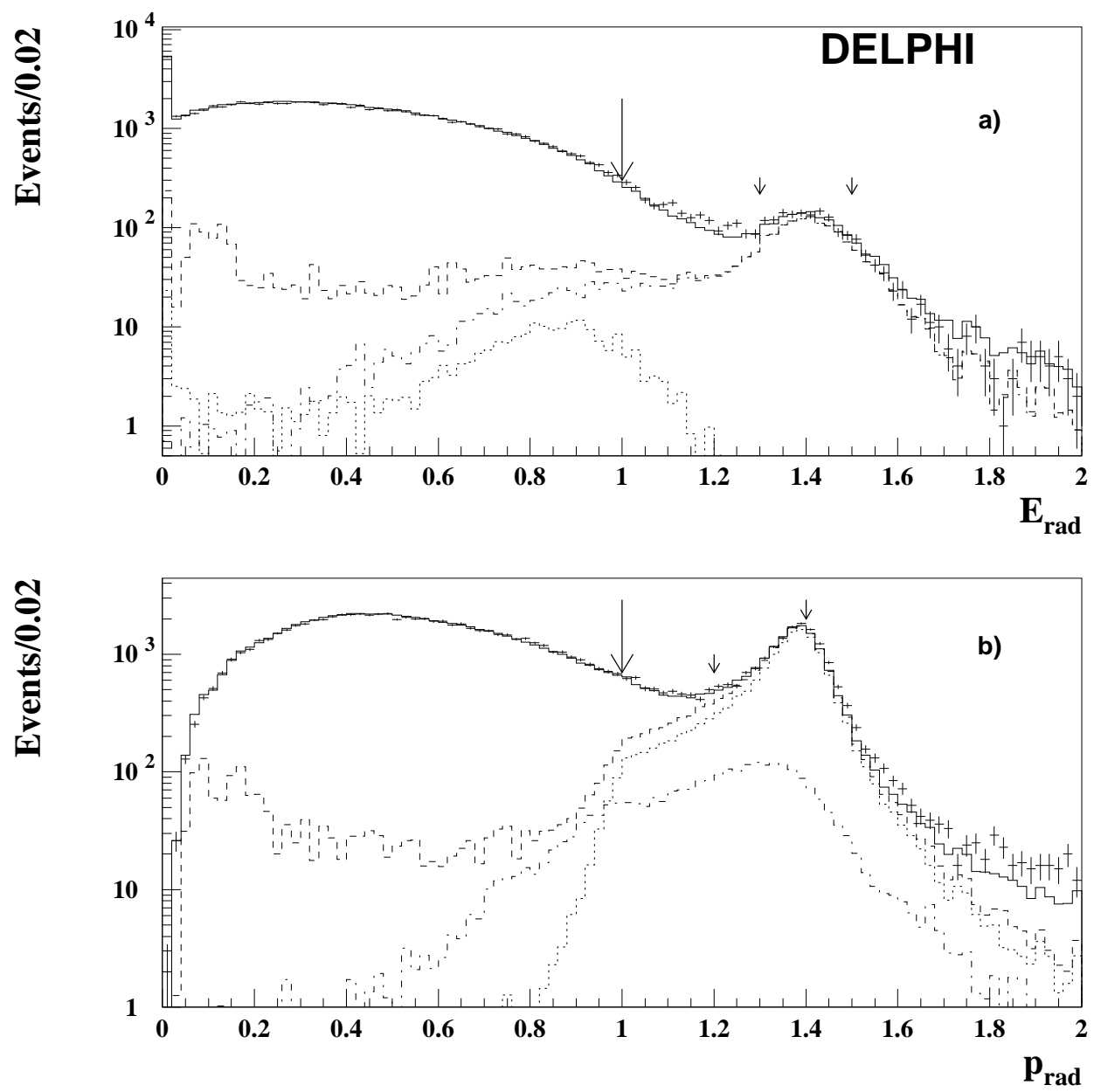

Figure 2: Distribution of a) $E_{\text {rad }}$ and b) $p_{\text {rad }}$ with no requirement on these variables. The figure shows the preselected $\tau^{+} \tau^{-}$sample used for electron identification. The crosses are for the data, the full line represents the expectation from the simulation. The dashed line shows the total background, with the contribution from $\mu^{+} \mu^{-}$and $\mathrm{e}^{+} \mathrm{e}^{-}$shown (dotted and dash-dotted respectively). Events to the left of the large arrows were retained as $\tau^{+} \tau^{-}$candidates. The two smaller arrows indicate the range used for normalisation of the background. 

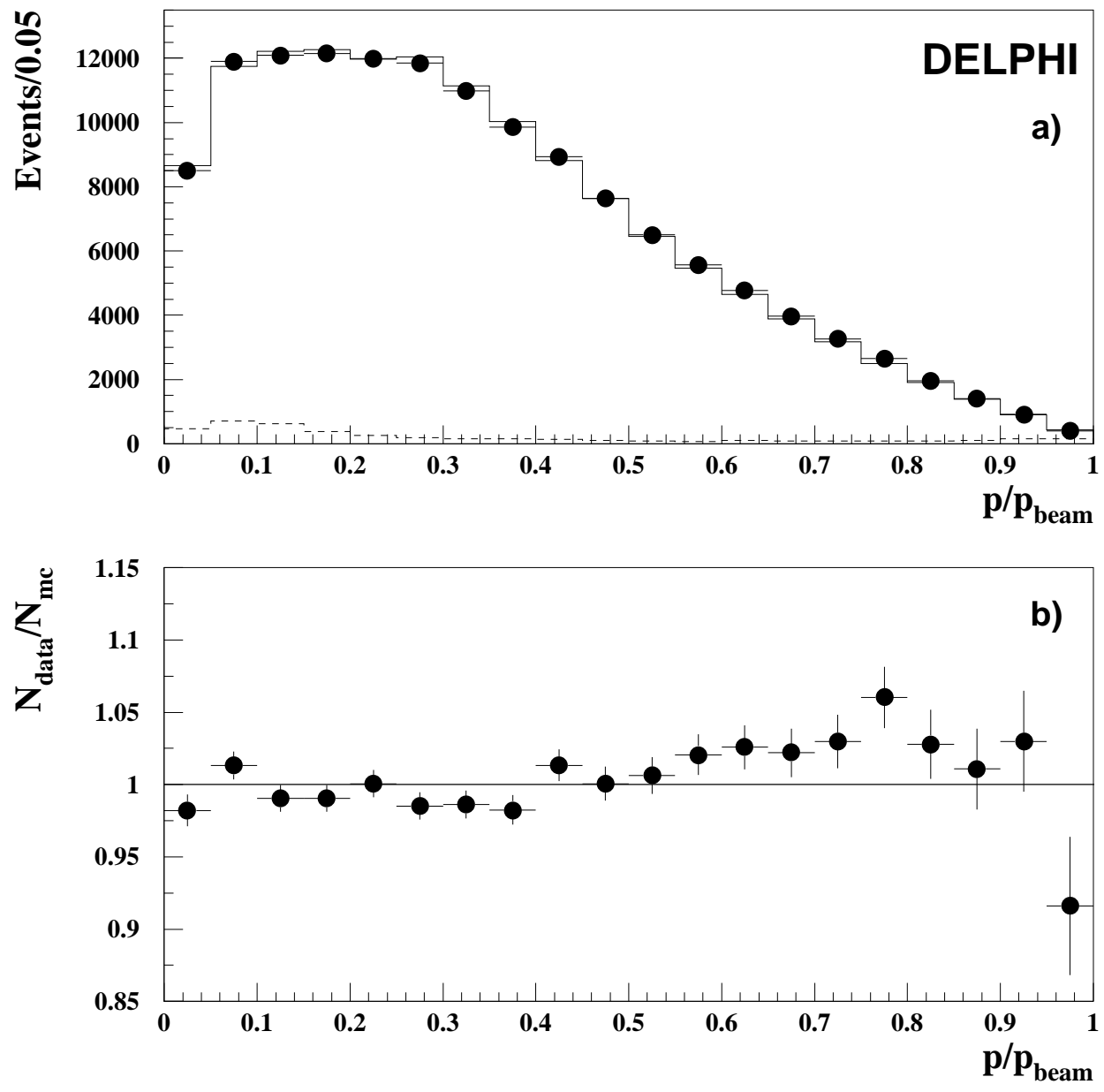

Figure 3: a) Distribution of the leading particle momentum for selected $\tau$ decays (points) compared to the expectation from simulation (line). The dashed line shows the expected background contribution. b) The ratio between data and simulation. The figure shows the preselected $\tau^{+} \tau^{-}$sample used for electron identification. 


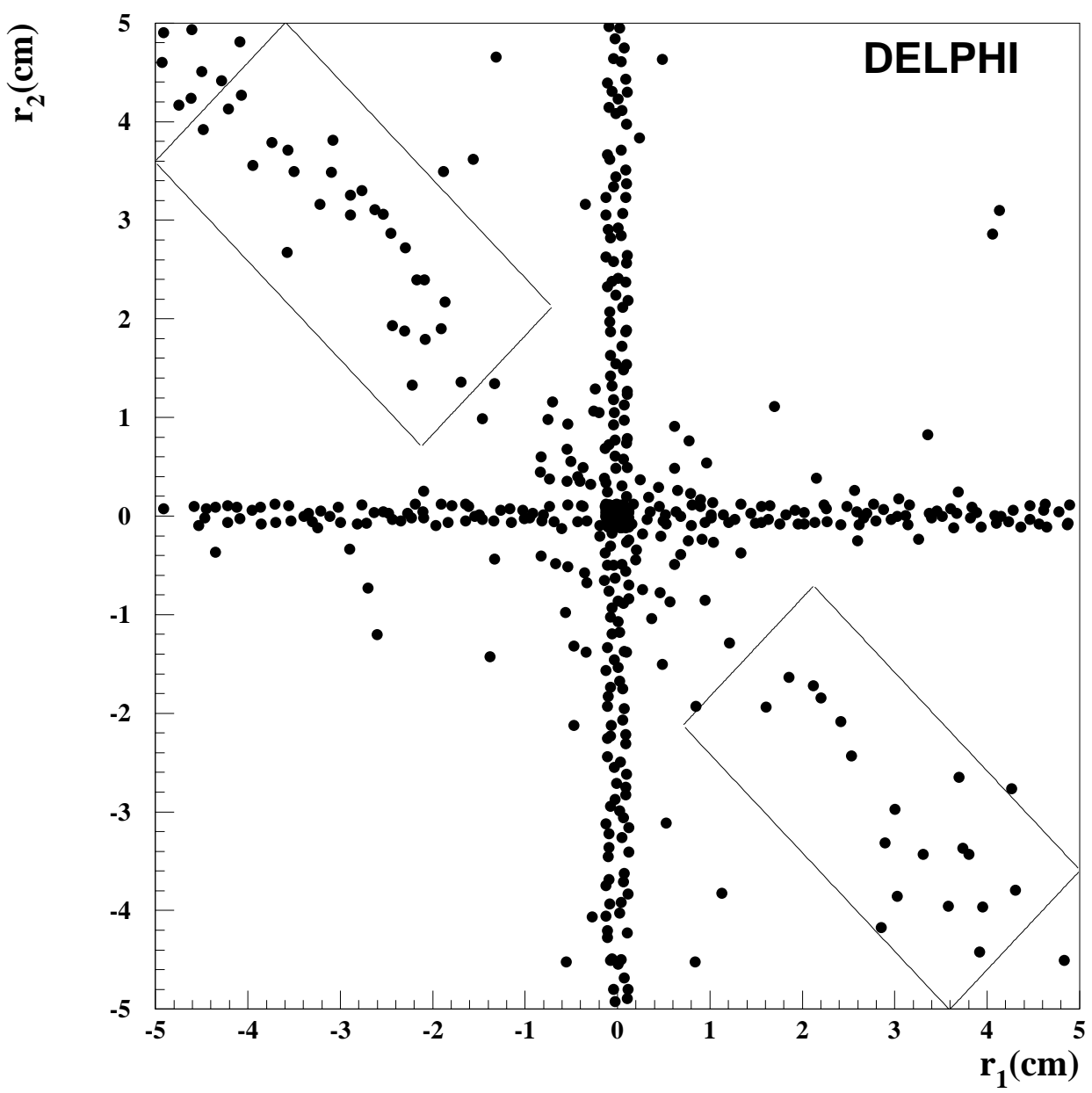

Figure 4: The impact parameter of the leading track in one hemisphere plotted against the impact parameter of the leading track in the other hemisphere. The number of events found inside the rectangles is used to estimate the number of cosmic ray events in the selected sample. 

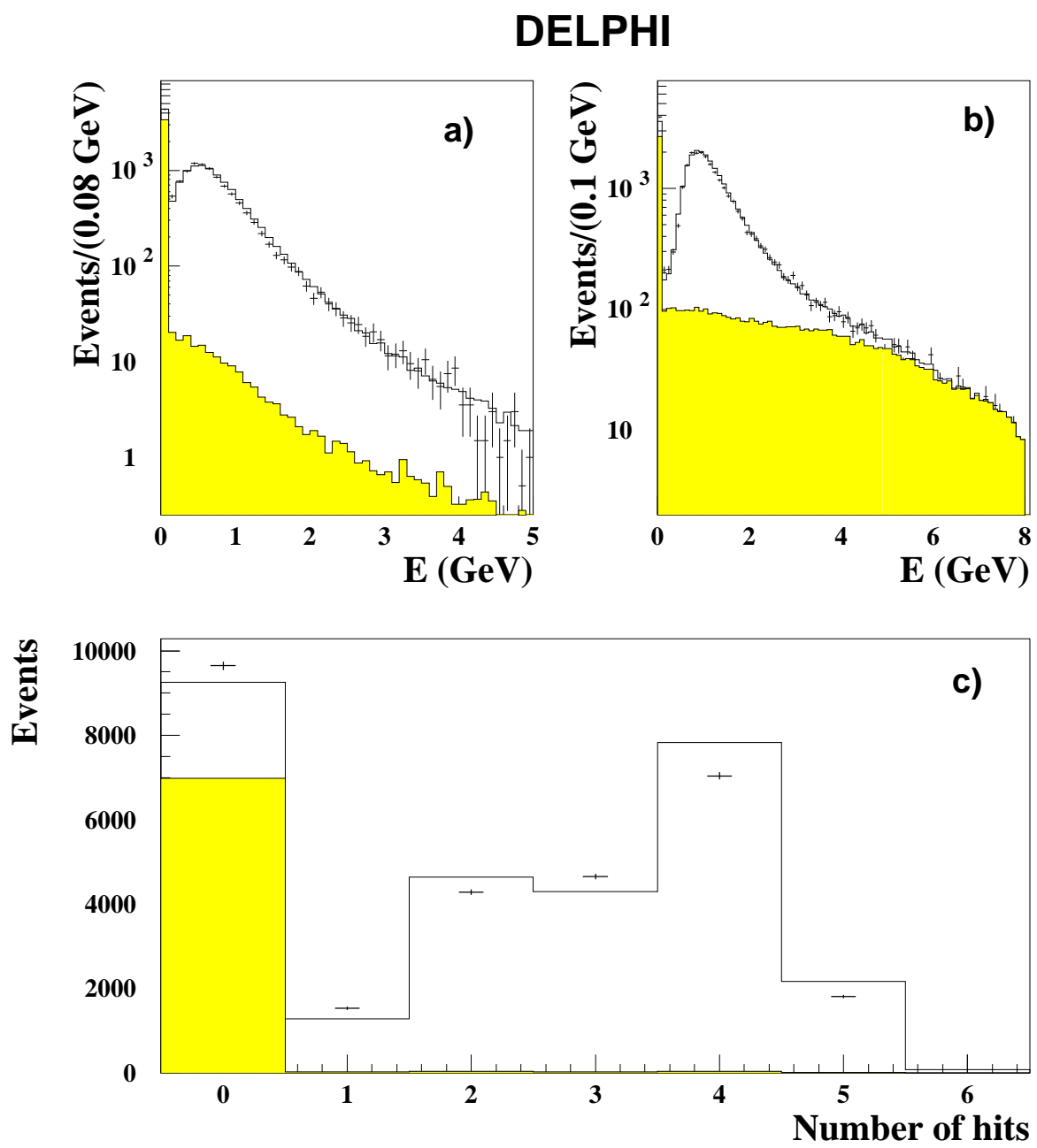

Figure 5: Muon identification variables: a) The energy of the outermost HCAL layer, b) largest HCAL energy deposition and c) the number of associated hits in the muon chambers. The crosses represent the data, the full line histograms represent the simulated events and the shaded areas are the non- $\mu$ contributions. All requirements described in the text were applied, except the one corresponding to the distributed variable. 


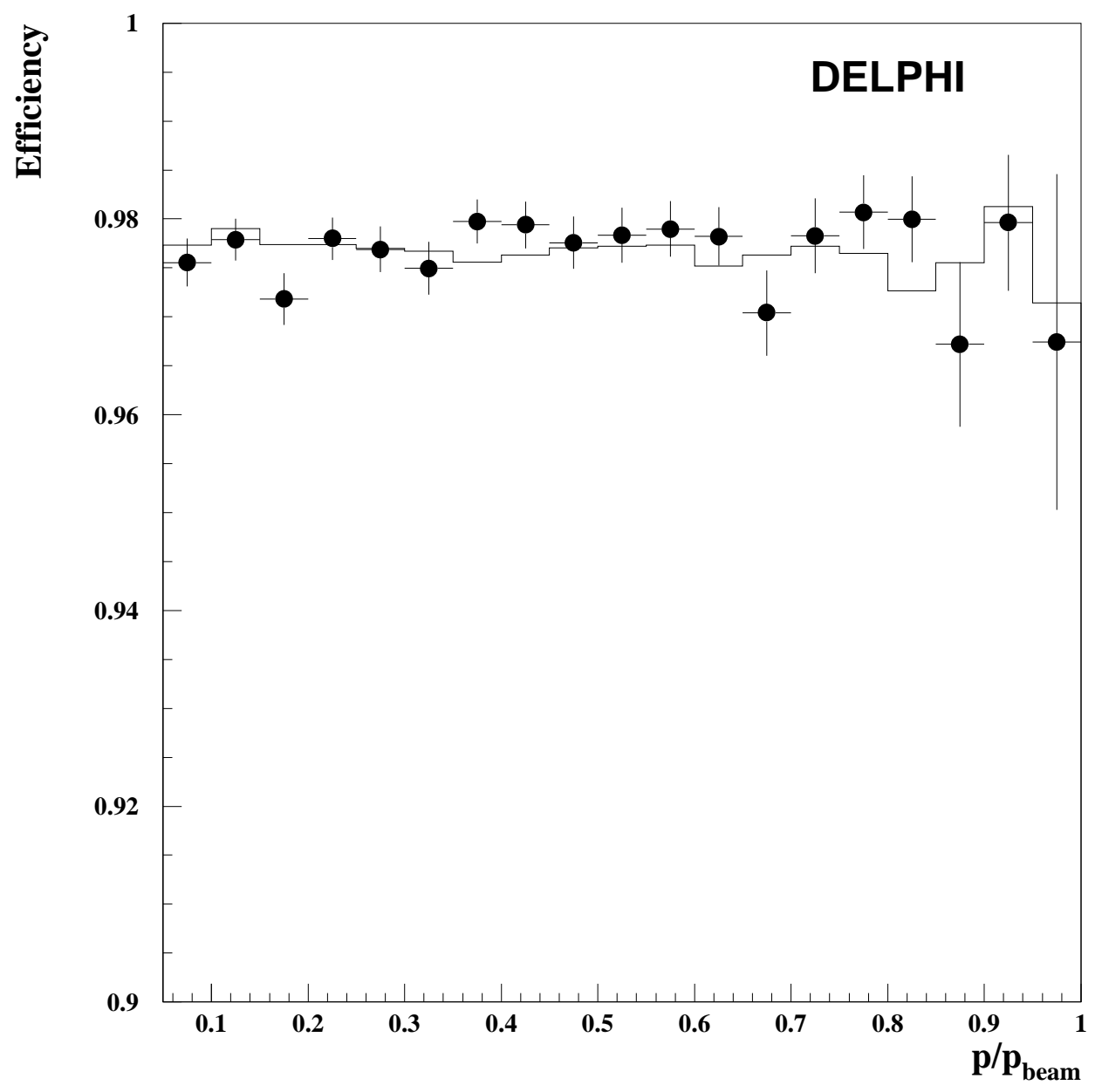

Figure 6: Comparison of data (points) and simulation (full line) for the estimate of the identification efficiency of the combined HCAL and muon chamber requirement. 

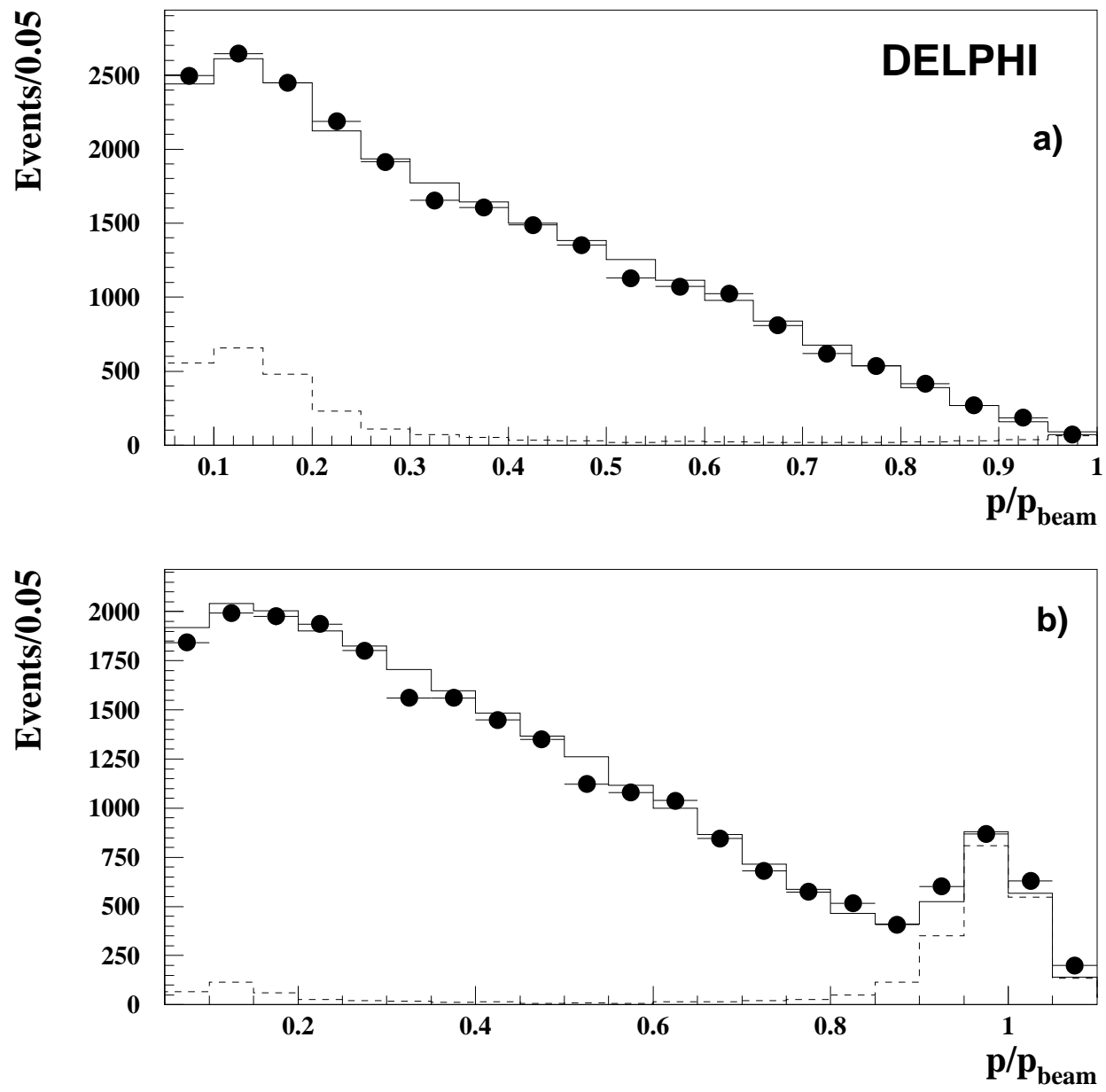

Figure 7: Momentum distribution of the sample of $\mu$ candidates when all the requirements of the analysis are applied except: a) the isolation angle criterion and b) the $p_{\text {rad }}$ requirement. The full line shows the expectation from simulation. The dashed line shows the expected background contamination. 

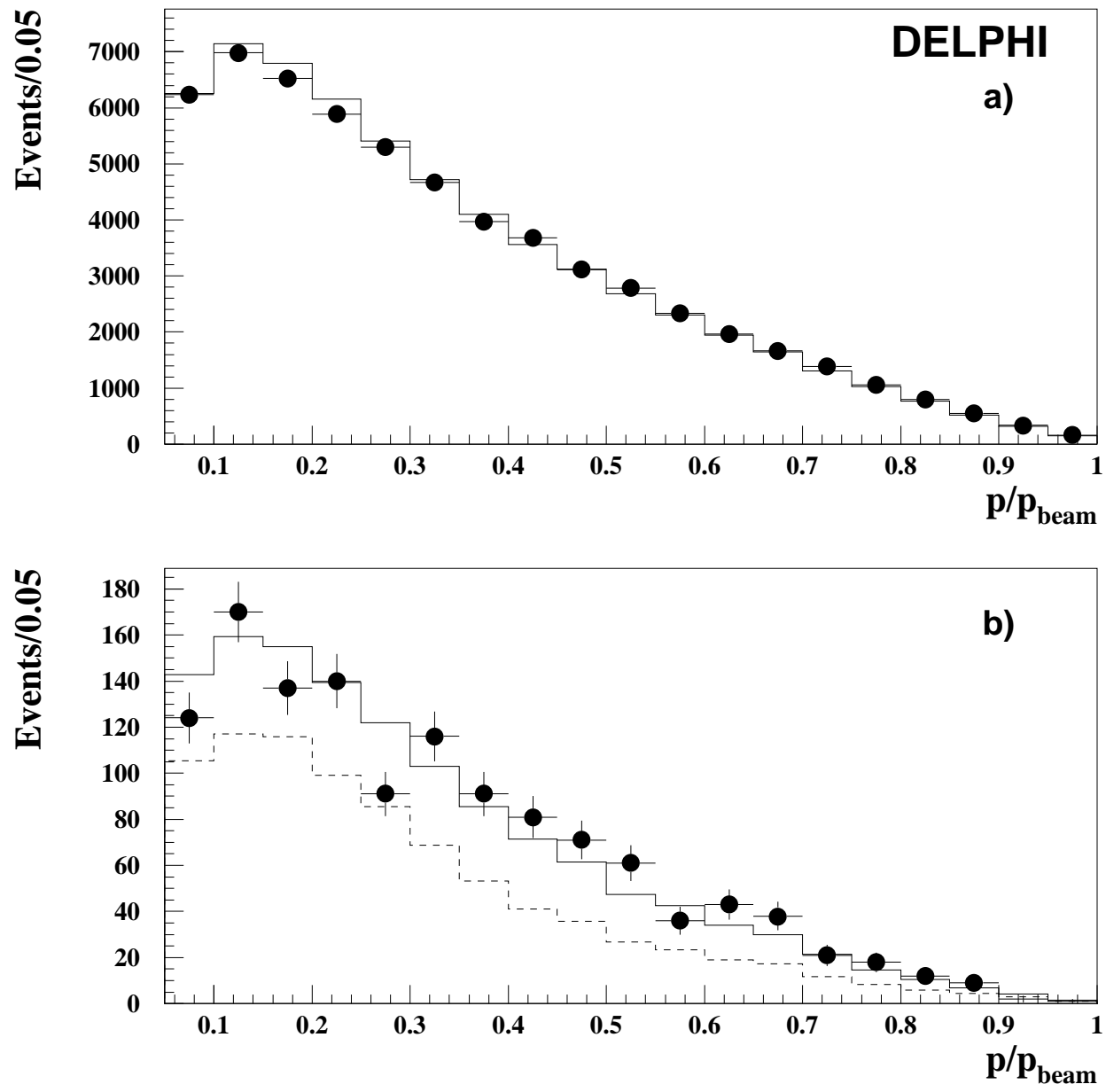

Figure 8: Momentum distribution of a sample of $\tau$ decay candidates strongly depleted in muons (see text): a) initial sample, b) remaining sample satisfying all muon identification requirement except the $E_{\text {cone }}$ requirement. The dashed line is the expected non-mu contribution to the distribution after an overall adjustment. The full line is the resulting expectation. 

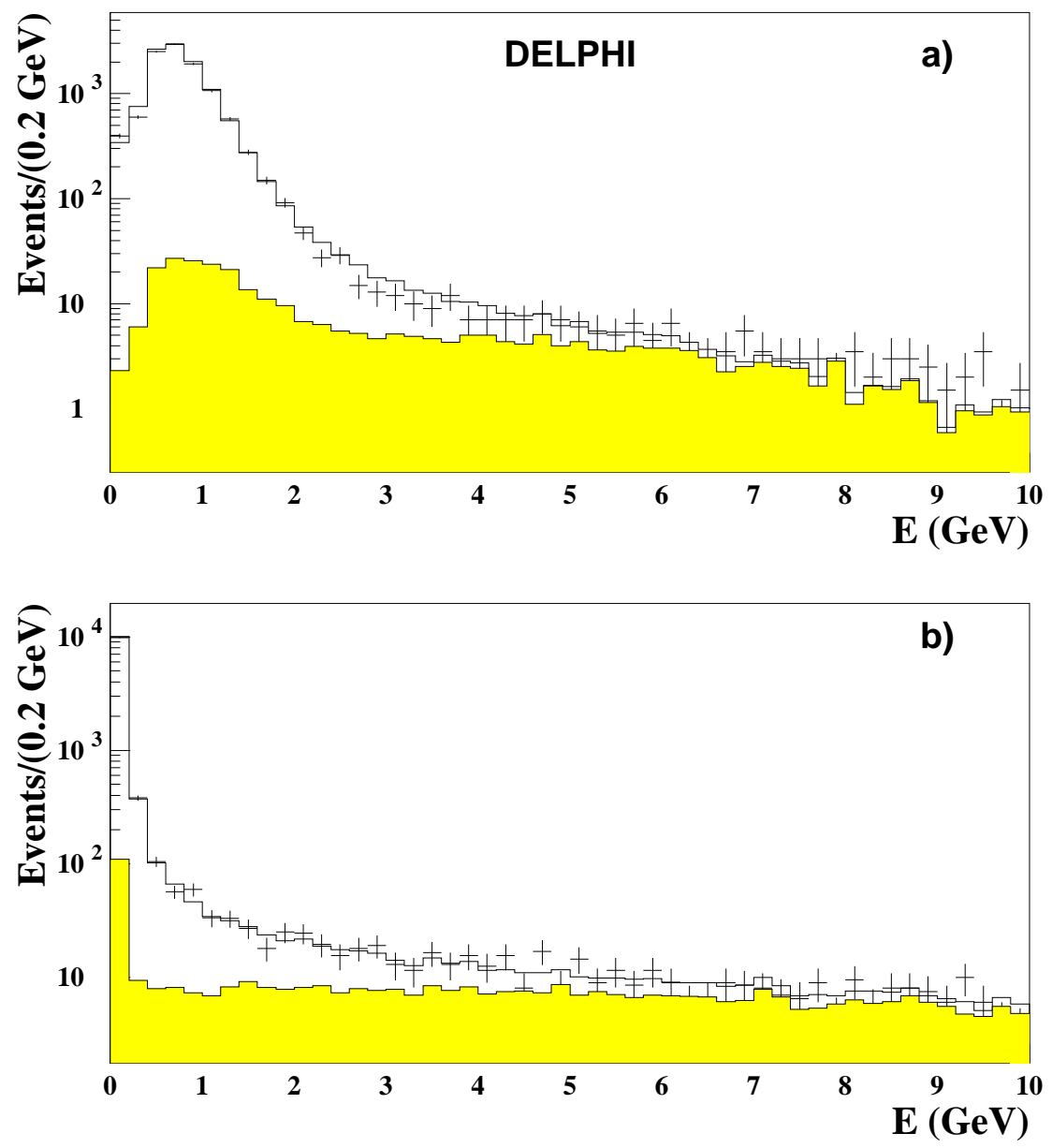

Figure 9: Distributions of a) the average energy deposition per layer in the HCAL, and b) the total electromagnetic energy in a cone with half opening angle of $18^{\circ}$ around the muon candidate track. The crosses show the data, while the full line shows the simulation result. The shaded area shows the contribution from backgrounds. 

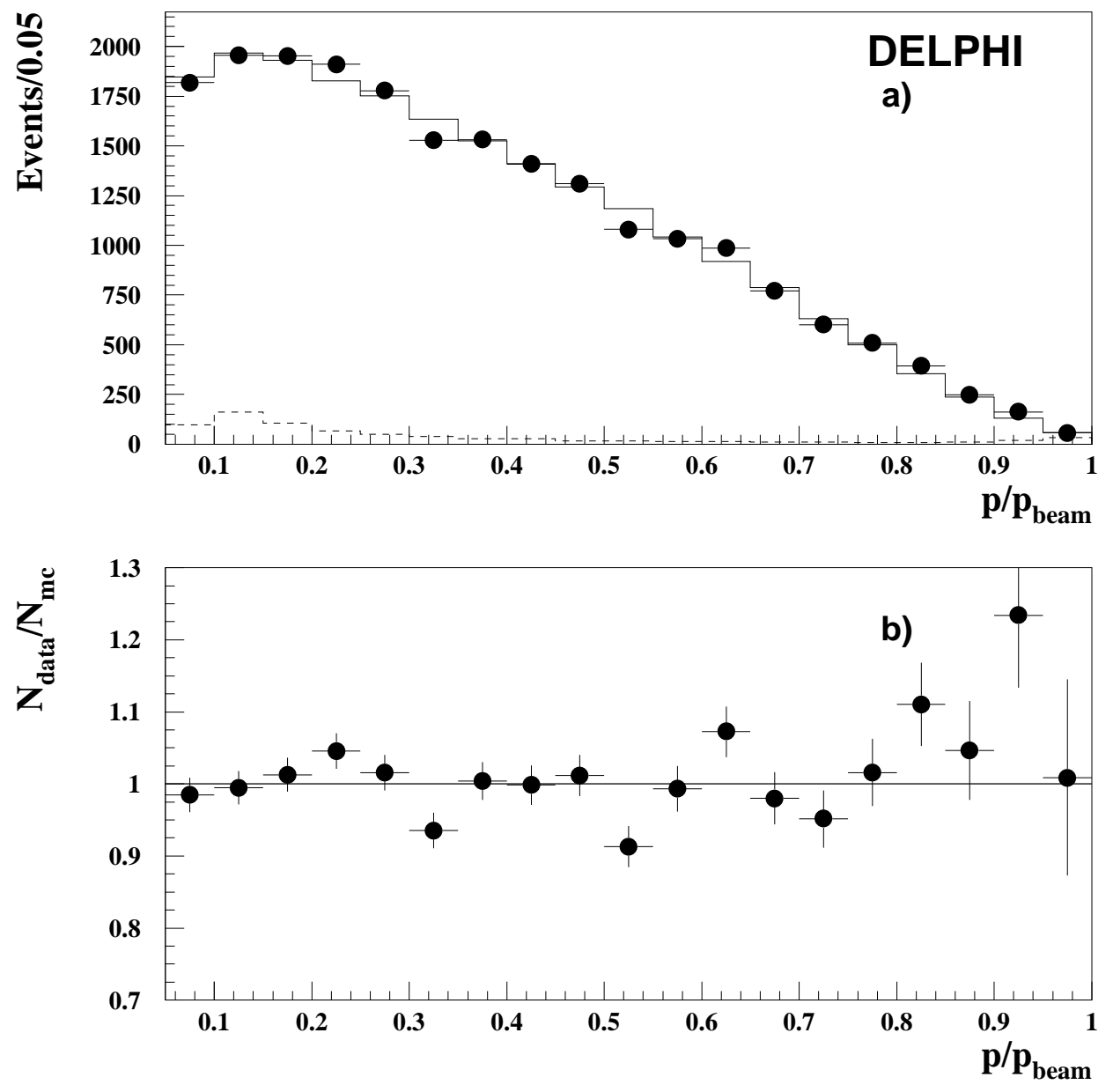

Figure 10: a) Momentum distribution of the sample of $\mu$ candidates. The points represent the data, the full line results from the simulated sample and the dashed line shows the background contribution. b) Ratio between data and simulation expectation as a function of the muon momentum. 

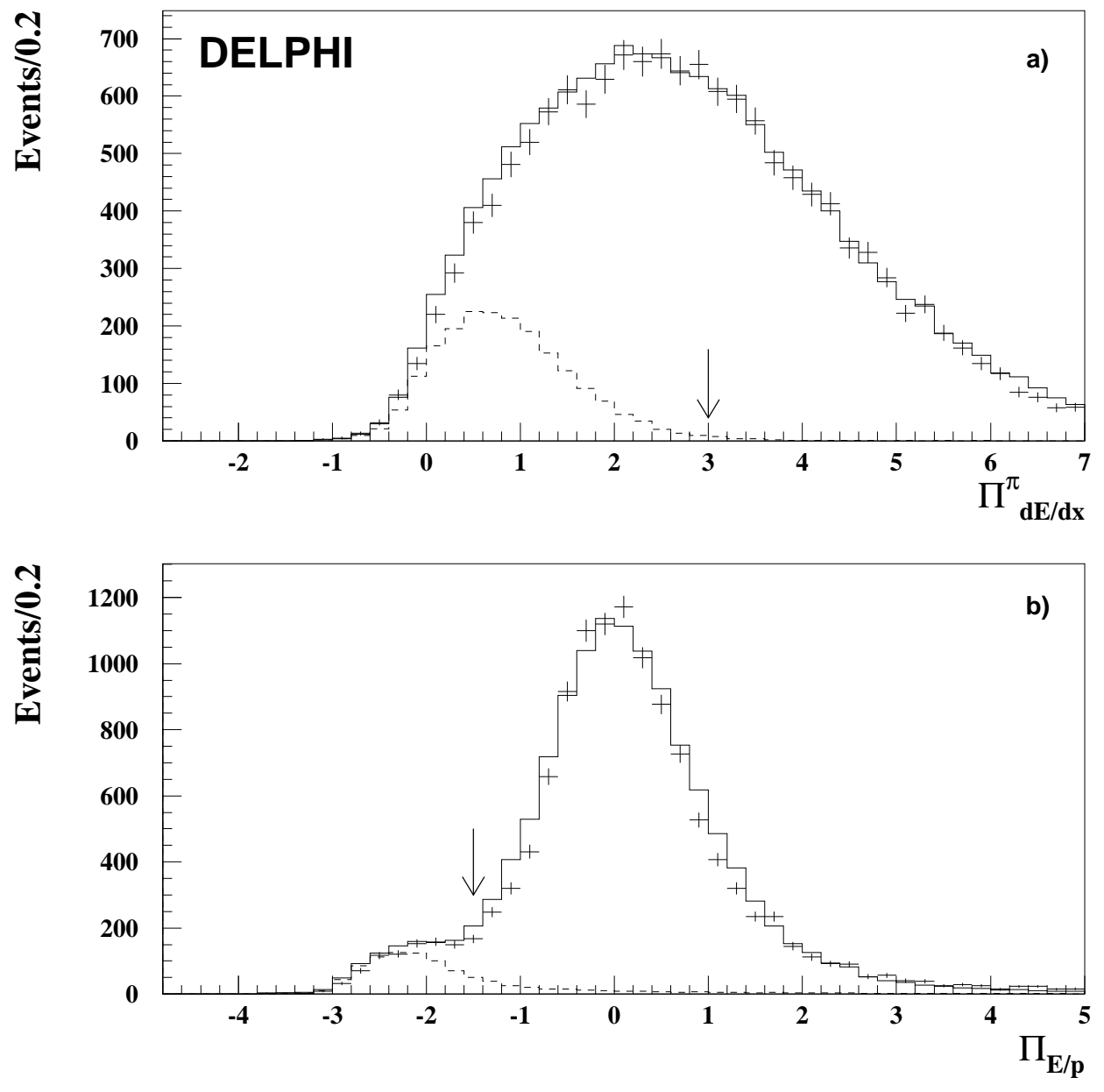

Figure 11: Distributions of variables used for electron identification in the momentum range $0.05<p / p_{\text {beam }}<0.5$ : a) $\Pi_{d E / d x}^{\pi}$, b) $\Pi_{E / P}$. Events are accepted for further analysis if at least one of the variables has a value above those indicated by the arrows. The points represent the data, the full line results from the simulated sample and the dashed line shows the background contribution. 

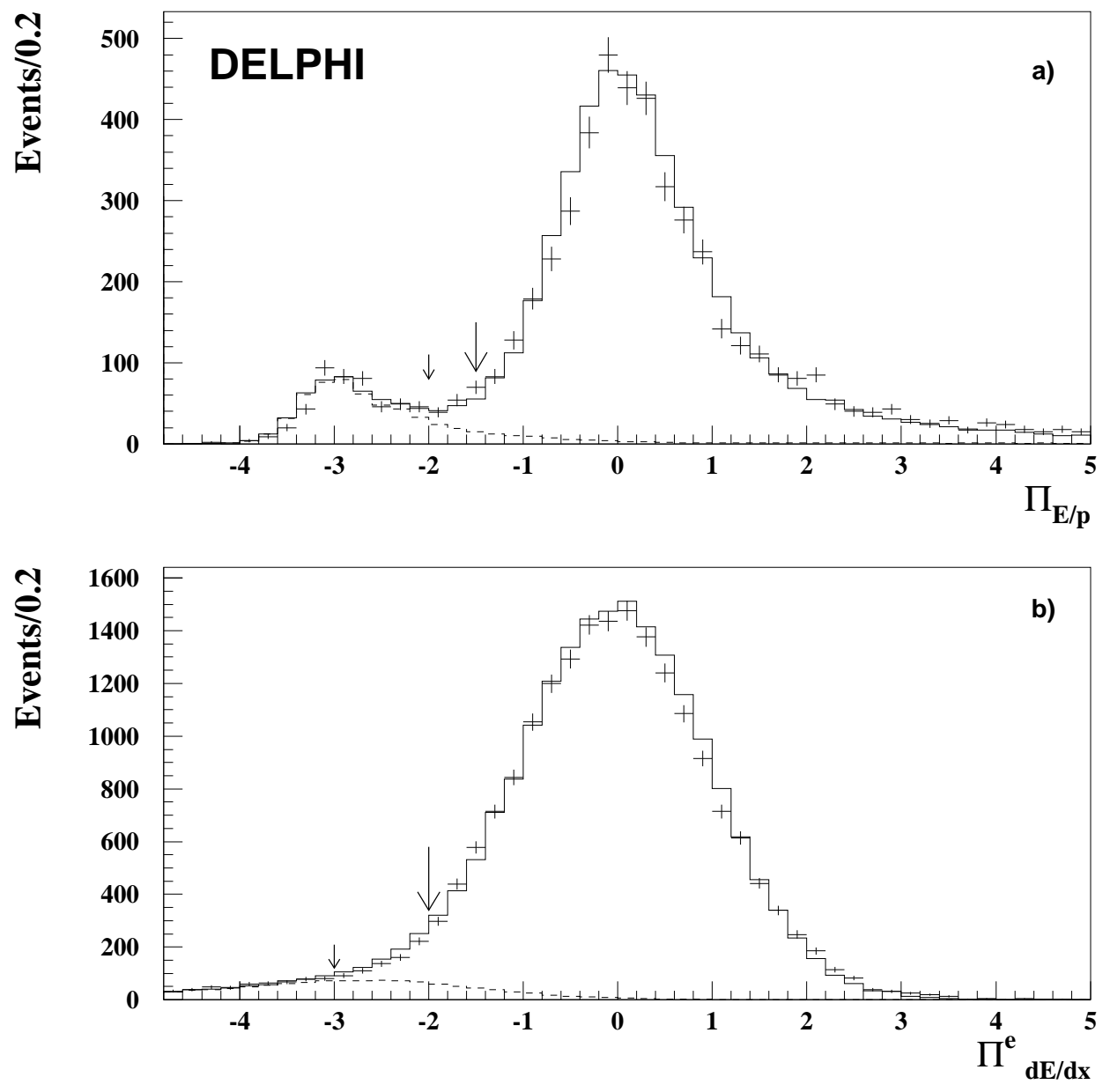

Figure 12: a) $\Pi_{E / P}$ for momenta above $0.5 \times p_{\text {beam }}$, b) $\Pi_{d E / d x}^{e}$ for the whole event sample. Events to the right of the large arrows are accepted as electron candidates. The background contribution is normalized to fit the number of events to the left of the small arrows. The points are data, the full line is the expectation from simulation, and the dashed line is the simulated background contribution. 

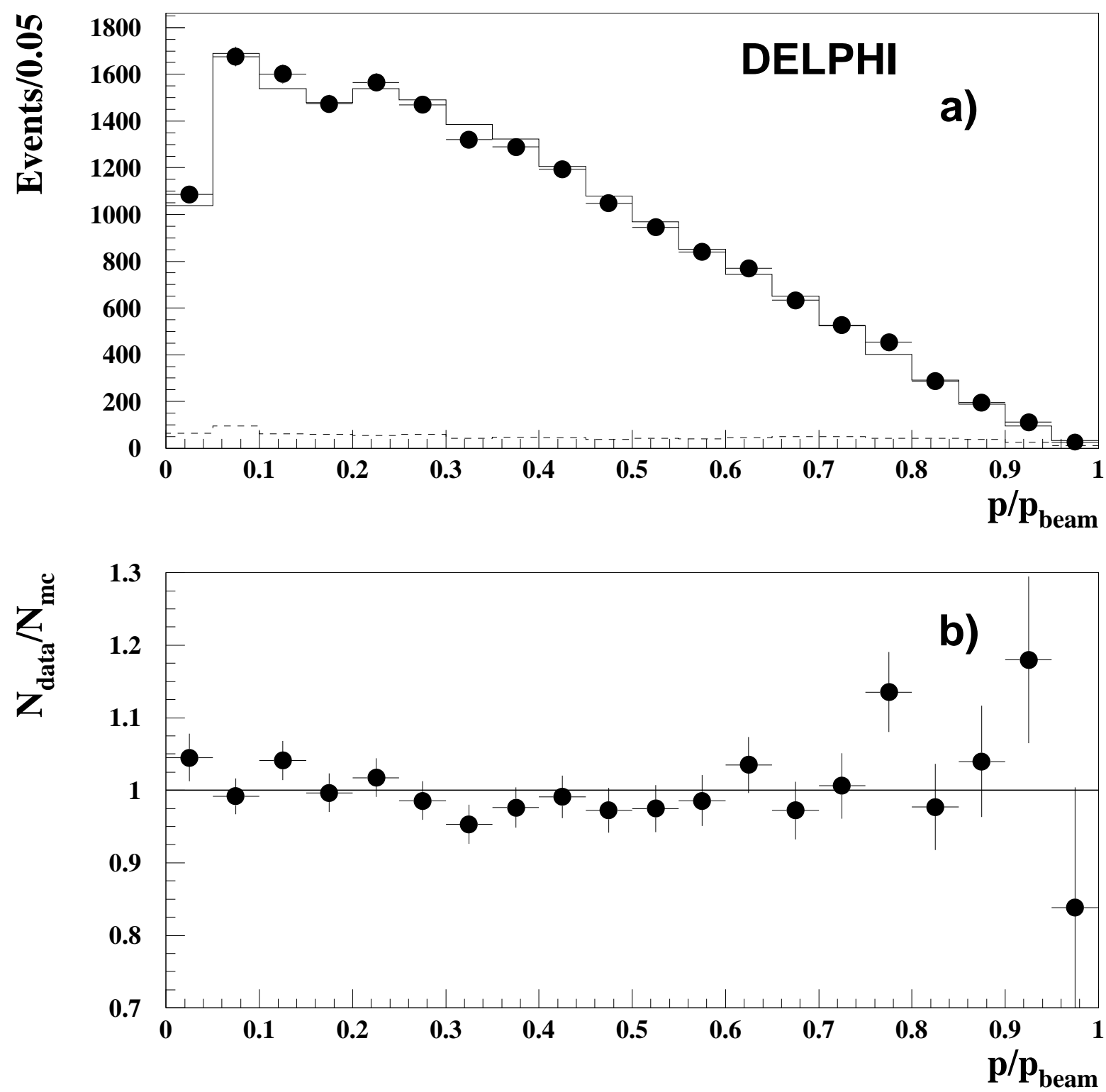

Figure 13: a) momentum distribution for identified electrons. The points are data, the full line is the expectation from simulation, and the dashed line is the simulated background contribution. b) The ratio between data and the expectation from simulation. 


\section{DELPHI}
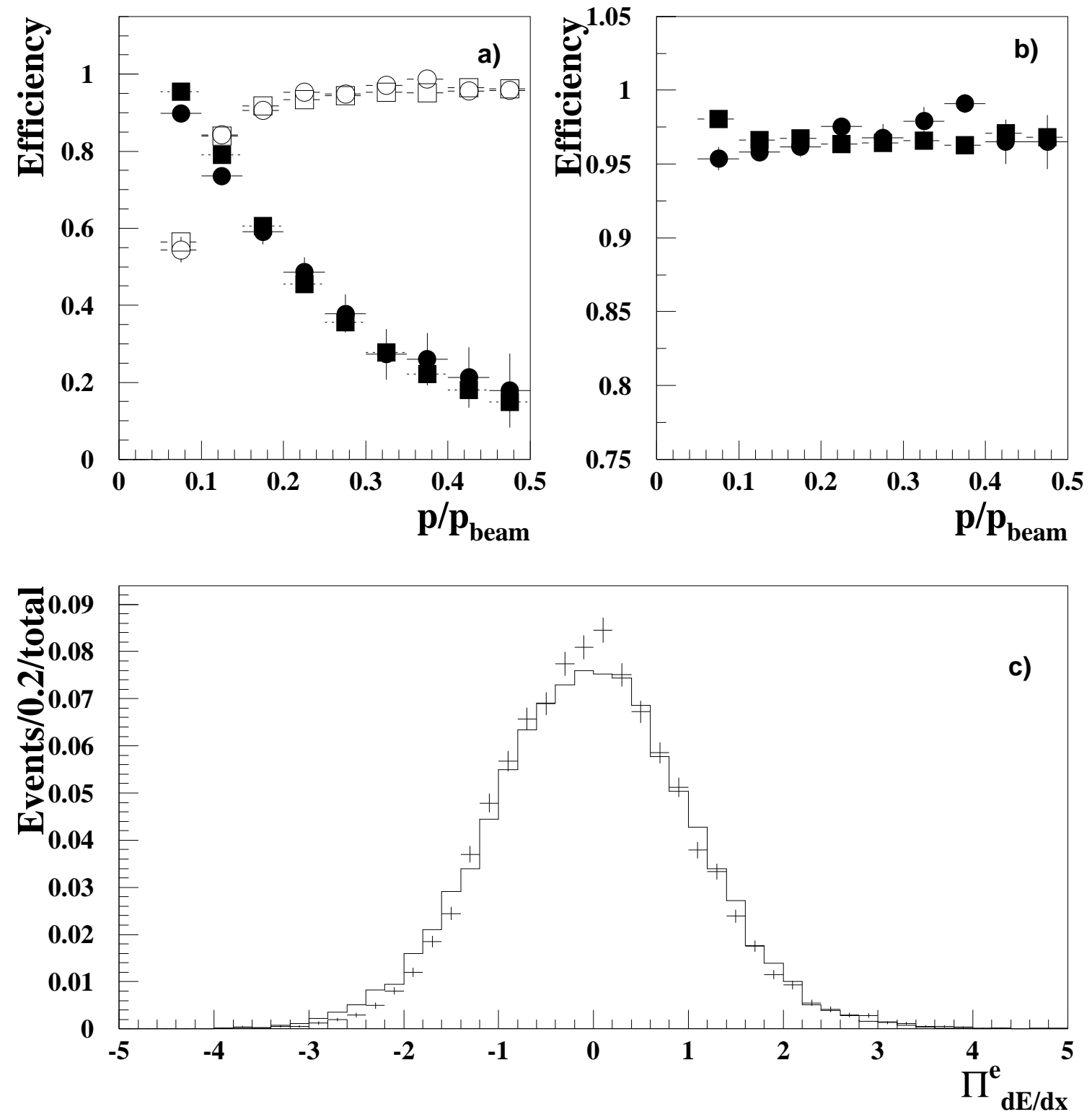

Figure 14: Example of efficiency checks, for the electron sample from 1994. a) Efficiency of the $\Pi_{E / p}$ (open marks) and $\Pi_{d E / d x}^{\pi}$ (solid marks) requirements as a function of momentum. b) The efficiency estimated from the logical sum (the '.OR.') of these requirements. The circles represent simulation, the squares are for data. c) $\Pi_{d E / d x}^{e}$ for a selected sample of Bhabhas. The crosses represent the data, the full line histogram corresponds to the simulation. 

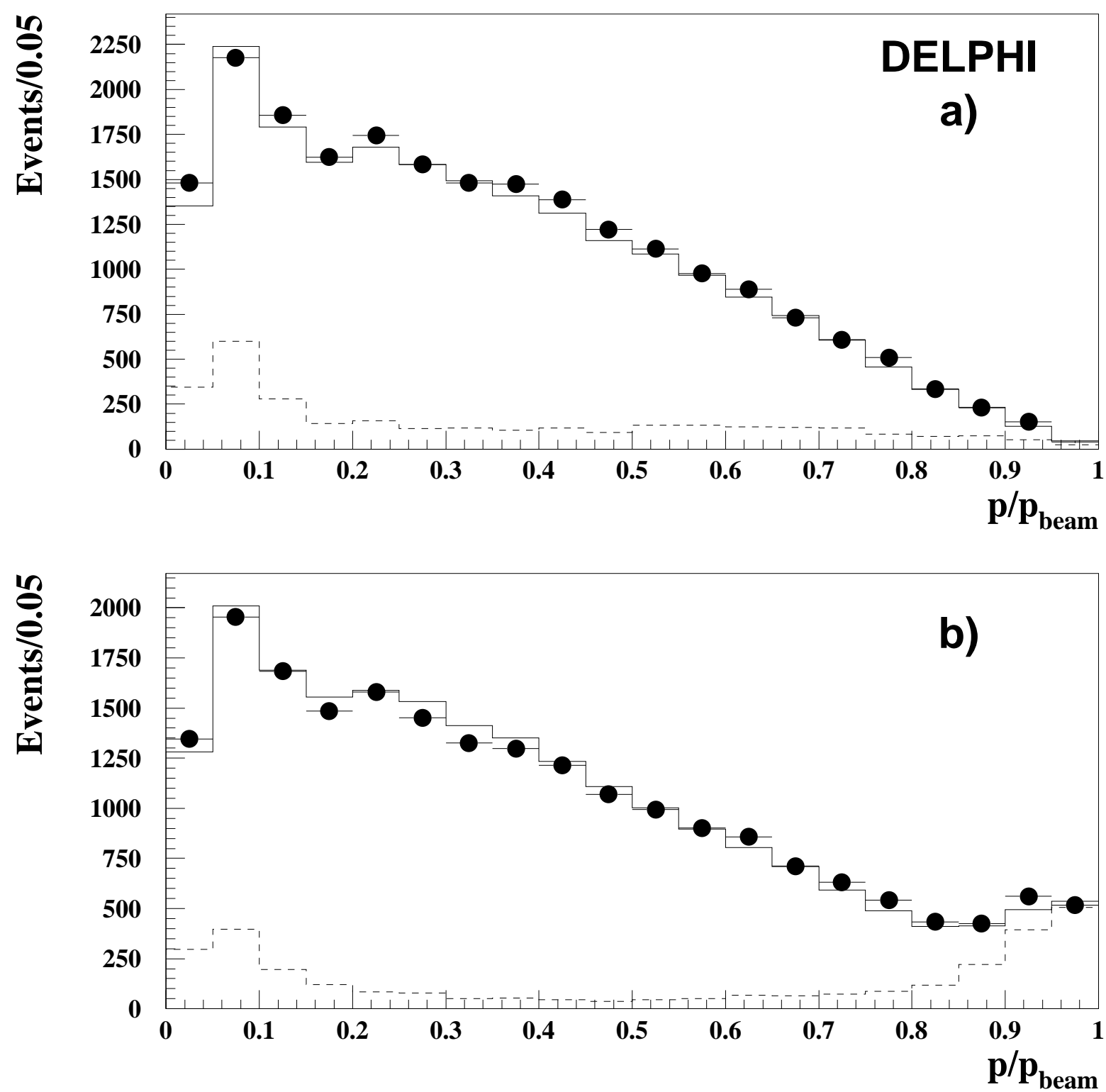

Figure 15: Momentum distribution for identified electrons with a) no isolation angle requirement and b) no $E_{\text {rad }}$ requirement. The points are data, the full line is the expectation from simulation and the dashed line is the simulated background contribution. 